ARTICLE

\title{
Disulfide-compatible phage-assisted continuous evolution in the periplasmic space
}

\author{
Mary S. Morrison 1,2,3, Tina Wang 1,2,3, Aditya Raguram¹,2,3, Colin Hemez (1) 1,2,3 \& David R. Liu (1) 1,2,3凶
}

The directed evolution of antibodies has yielded important research tools and human therapeutics. The dependence of many antibodies on disulfide bonds for stability has limited the application of continuous evolution technologies to antibodies and other disulfidecontaining proteins. Here we describe periplasmic phage-assisted continuous evolution (pPACE), a system for continuous evolution of protein-protein interactions in the disulfidecompatible environment of the $E$. coli periplasm. We first apply pPACE to rapidly evolve novel noncovalent and covalent interactions between subunits of homodimeric YibK protein and to correct a binding-defective mutant of the anti-GCN4 $\Omega$-graft antibody. We develop an intein-mediated system to select for soluble periplasmic expression in PPACE, leading to an eight-fold increase in soluble expression of the $\Omega$-graft antibody. Finally, we evolve disulfide-containing trastuzumab antibody variants with improved binding to a Her2-like peptide and improved soluble expression. Together, these results demonstrate that PPACE can rapidly optimize proteins containing disulfide bonds, broadening the applicability of continuous evolution.

\footnotetext{
${ }^{1}$ Merkin Institute of Transformative Technologies in Health Care, Broad Institute of Harvard and MIT, Cambridge, MA 02142, USA. ${ }^{2}$ Department of Chemistry and Chemical Biology, Harvard University, Cambridge, MA 02138, USA. ${ }^{3}$ Howard Hughes Medical Institute, Harvard University, Cambridge, MA

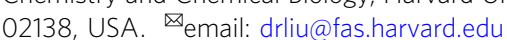


A ntibodies and their engineered derivatives are important treatments for diverse inflammatory, autoimmune, and infectious diseases, as well as many cancers, including HER2-positive breast cancer, non-Hodgkin's lymphoma, and melanoma ${ }^{1}$. Monoclonal antibodies (mAbs) and their derivatives now represent the largest class of therapeutic protein drugs, with 82 therapeutic antibodies currently approved by the FDA and hundreds in clinical trials ${ }^{2}$.

Antibody-based therapies are limited by high development costs $^{1,3}$. Directed evolution has the potential to decrease cost and accelerate the development of novel and potent antibodies. While multiple selection systems have been shown to evolve new antibody-antigen interactions in E. coli, including phage display ${ }^{4-7}$, $\mathrm{APEx}^{8}, \mathrm{FLI}-\mathrm{TRAP}^{9}, \mathrm{cyclonal}^{10}, \mathrm{BAD}^{11}$, inner-membrane display ${ }^{12}$, and $\mathrm{AHEAD}^{13}$, many of these techniques require researcher intervention to carry out time-intensive steps. Continuous selection platforms, in which all stages of the evolutionary cycle are carried out by automated or in vivo processes without the need for researcher invention, have the potential to substantially streamline antibody development ${ }^{4-27}$.

Phage-assisted continuous evolution (PACE) is a rapid directed evolution system capable of evolving proteins over days or weeks, with minimal human intervention during evolution ${ }^{28}$. In PACE, an evolving protein is encoded in place of gene III (gIII) in the genome of M13 bacteriophage (Fig. 1a). An accessory plasmid (AP) within a host $E$. coli cell expresses gIII in response to the desired function of the evolving protein. As phage depends on PIII, the protein product of gIII, to efficiently infect host cells, PACE links the desired property of an evolving protein with phage fitness ${ }^{28}$. Phage propagates in a fixed-volume vessel (the "lagoon") that is diluted with a constant inflow of new host cells from a population maintained in a chemostat. Phage that fails to propagate is quickly washed out of the lagoon by inflowing cells. Selection pressure is controlled by moderating the flow rate, and by modifying the genetic circuit governing gIII expression on the AP. Inducible mutagenesis plasmids (MPs) elevate the error rate of DNA replication when induced, allowing simultaneous selection and mutagenesis ${ }^{28,29}$. One complete generation of evolution occurs with each phage reproductive cycle $(\sim 10 \mathrm{~min} \text { to } 1 \mathrm{~h})^{28}$.

PACE has been used to evolve diverse classes of proteins, including polymerases $^{19,30}$, proteases $^{31-33}$, tRNA synthetases ${ }^{34}$, agricultural toxins ${ }^{28}$, TALENs ${ }^{35}$, Cas9 variants ${ }^{36,37}$, dehydrogenases ${ }^{38}$, deaminase enzymes $^{39}$, antibody fragments ${ }^{39}$, cytosine base editors ${ }^{40}$, adenine base editors ${ }^{41}$ and biosynthetic pathways ${ }^{42}$. However, continuous in vivo evolution platforms, including PACE, have thus far been limited to the cytoplasm of the host cell ${ }^{19,23-28,30-42}$. Confining selections to the cytoplasm maintains the linkage between genotype and phenotype. In both prokaryotes and eukaryotes, however, the cytoplasm is a chemically reducing environment, and does not support the formation of cysteine disulfide linkages. Disulfide bonds are crucial determinants of stability and proper folding for many proteins, including antibodies and antibody fragments. Loss of stabilizing disulfide bonds often leads to aggregation during cytoplasmic expression $^{43}$, making disulfide-enriched proteins a challenging class of proteins to evolve by currently available continuous directed evolution techniques ${ }^{11,39,44-49}$.

Disulfide bond formation can be supported in the cytosol through the expression of thiol oxidase and disulfide isomerase in the cytoplasmic space ${ }^{50}$, but introducing non-native oxidative chemistry into the bacterial cytoplasm increases cellular stress, membrane impairment and aggregation ${ }^{51}$, a hurdle for the continuous-flow and liquid-handling devices used in continuous directed evolution. Alternatively, directed evolution can be applied to compensate for the loss of disulfides, but this process adds complexity and steps that are not ultimately necessary in proteins intended for use outside the cell. Compensatory stabilizing mutations may also result in trade-off costs to target affinity $39,45-49$, limiting the scope and relevance of the resulting proteins for use outside of cells. Finally, binding affinity evolutions in the cytoplasm are not compatible with disulfides in the target protein, excluding many extracellular antigens of therapeutic interest. It is thus more biologically relevant to evolve disulfide-containing proteins in oxidizing environments if the protein is intended for extracellular use.

The bacterial periplasm is an oxidizing environment that supports the formation of disulfides in proteins such as antibodies ${ }^{43,52}$. Expression of evolving proteins in the periplasm permits disulfide bond formation while retaining the evolving protein within the bacterial host cell.

In this study, we demonstrate a PACE system for the continuous evolution of proteins in the periplasmic space. This platform supports the formation of disulfide bonds in the evolving protein of interest and represents, to our knowledge, the first application of PACE to interactions occurring in a cellular compartment other than the cytoplasm and the first continuous in vivo evolution of proteins under oxidizing conditions. Periplasmic PACE (pPACE) can be tuned to select for enhanced soluble expression in addition to enhanced binding activity.

We validate $\mathrm{pPACE}$ by restoring binding in the homodimeric protein YibK and in the $\Omega$-graft scFv. We apply pPACE to evolve a minimized form of the antibody-drug trastuzumab (Herceptin), achieving up to 2.5-fold improved binding of a Her2-mimetic peptide and 6-fold increased soluble expression, without any loss of native Her2 affinity. Together, these results establish pPACE as a method that substantially expands the scope of continuous protein evolution to include proteins that require a non-reducing environment to fold or function.

\section{Results}

CadC activates transcription upon periplasmic binding. A periplasmic protein-protein interaction selection system must convert a periplasmic binding event into a cytoplasmic transcriptional activation event. We examined transmembrane signaling proteins that physically link protein-protein binding in the periplasm with transcription in the cytoplasm. CadC is a native $E$. coli sensor protein and a member of the ToxR-like receptor family. Several members of the ToxR family have served as the basis for engineered sensors of periplasmic or transmembrane interactions ${ }^{53-55}$. CadC consists of a periplasmic sensor domain, a transmembrane helix, and a DNA-binding cytoplasmic domain (Fig. 1b). Under stress conditions, the periplasmic sensor domains from two CadC molecules homodimerize, bringing together the cytoplasmic DNA-binding domains to generate two cooperative DNA-binding sites, which then bind two DNA motifs, Cad1 and Cad2, on the CadBA promoter $\left(\mathrm{P}_{\text {cadBA }}\right)$ to initiate gene transcription ${ }^{54,56}$. Replacement of the sensor domain with a dimerizing protein leads to constitutive activation of $\mathrm{P}_{\text {cadBA }}{ }^{53}$. CadC thus converts binding in the periplasm into cytoplasmic transcriptional activation.

We reasoned that CadC could form the basis of a PACE selection for protein-protein binding in the periplasmic space (Fig. 1c). First, we optimized $\mathrm{P}_{\text {cadBA }}$ (Supplementary Fig. 1) and deleted the host genomic cadCBA operon to minimize background transcription (Supplementary Fig. 2). To validate that protein binding could trigger transcription at $\mathrm{P}_{\text {cadBA }}$, we expressed CadC with its sensory domain replaced by the HA4 monobody, a high-affinity monobody that binds the $\mathrm{SH} 2$ domain of ABL1 kinase ${ }^{57}$. We then expressed YibK, a homodimeric knottin protein, fused to the $\mathrm{SH} 2$ binding target of HA4. This construct was directed to the periplasm by an $\mathrm{N}$-terminal signal sequence (SS) peptide derived from alkaline 
a

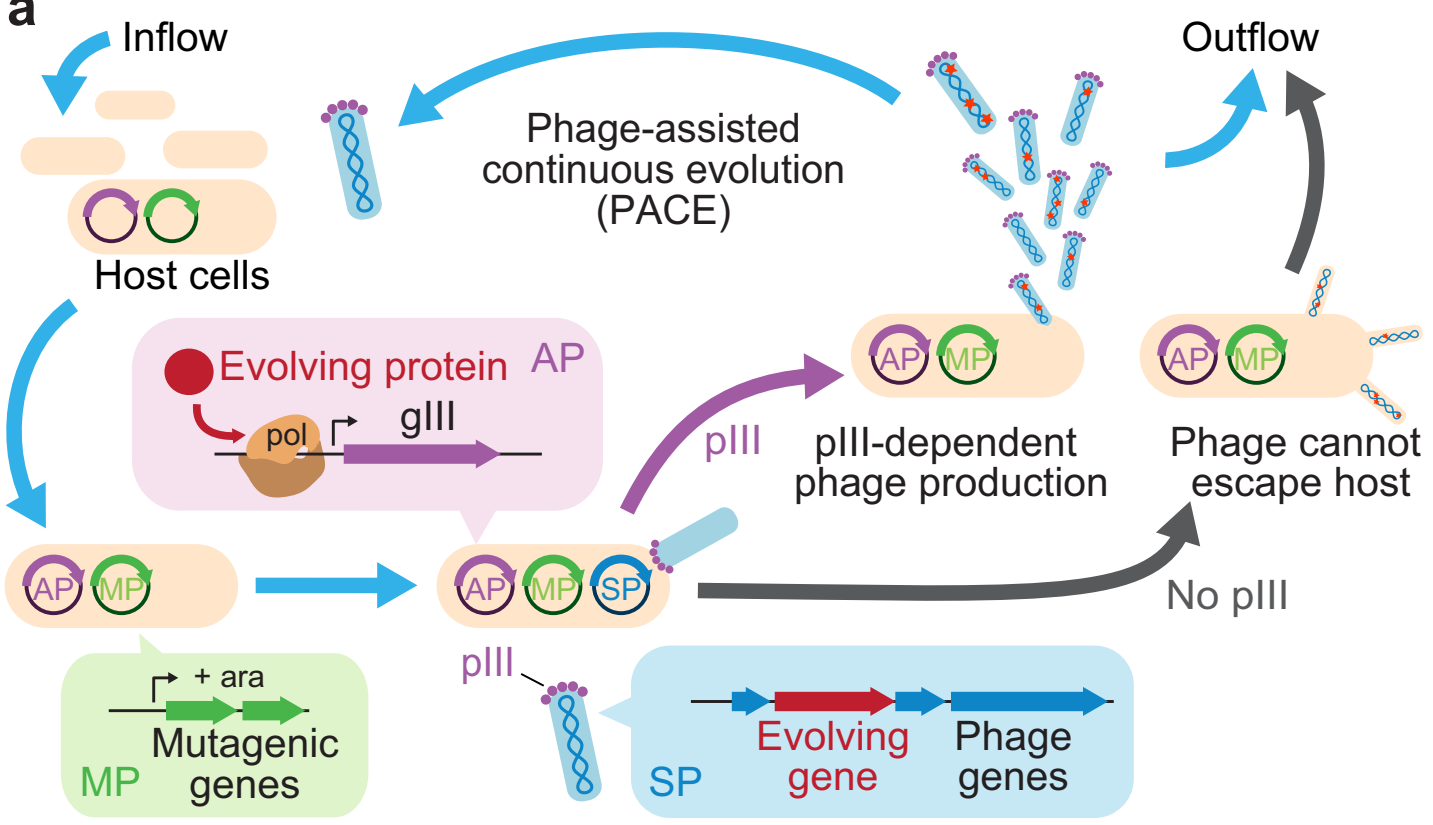

b
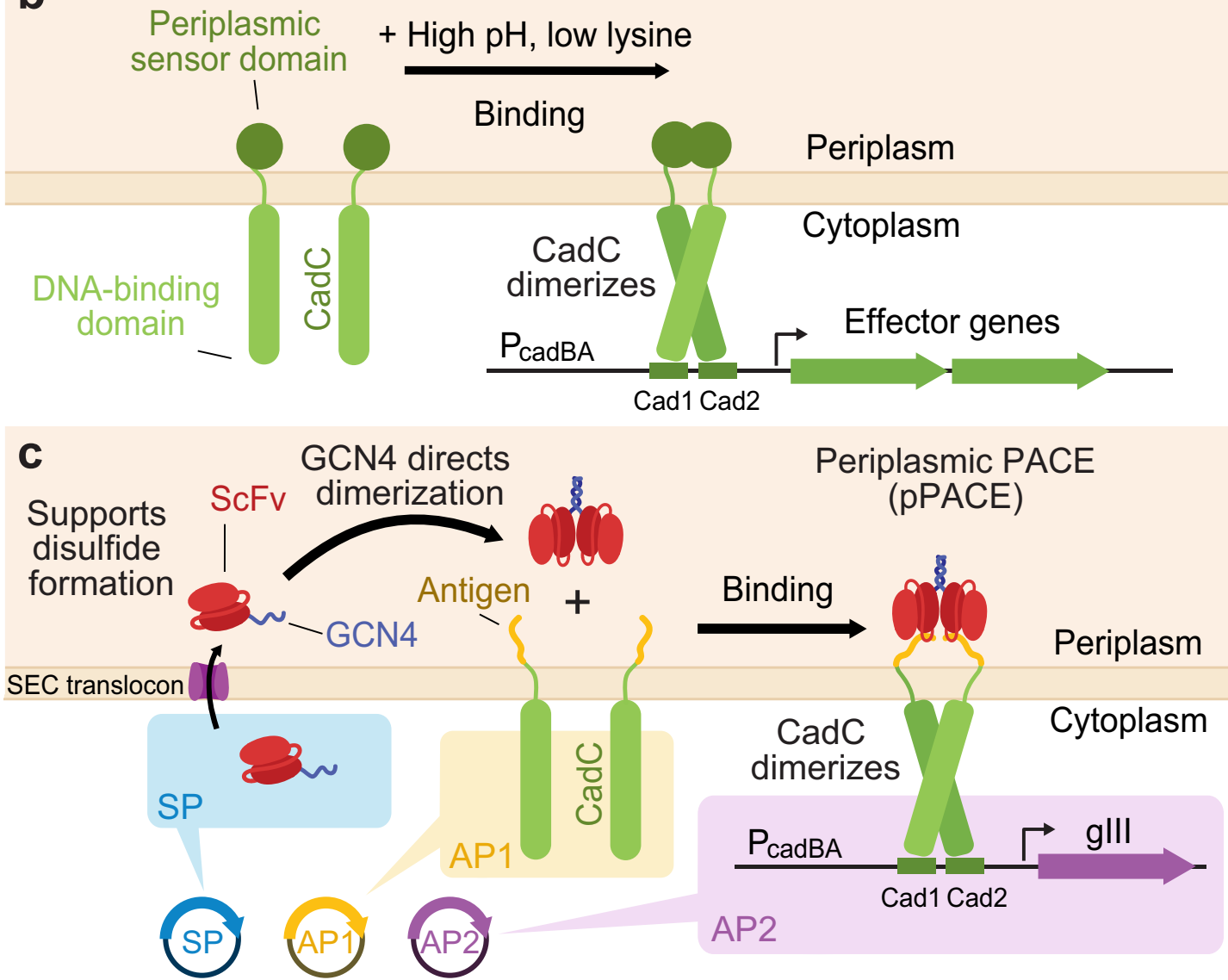

phosphatase A (PhoA), ${ }^{58}$. YibK homodimerization should trigger dimerization of the CadC-HA4 fusion via binding of HA4 to the $\mathrm{SH} 2$ domain fused to YibK, resulting in activation of $\mathrm{P}_{\text {cadBA. }}$. Indeed, we observed that expression of SS-YibK-SH2 directed $\mathrm{P}_{\text {cadBa }}$ transcriptional activation 66-fold over expression of cytoplasmic YibK-SH2 as measured by transcription assay (Fig. 2b).
The point mutation V139R blocks YibK dimerization by disrupting hydrophobic interactions between YibK monomers and preventing a final folding transition to the native YibK structure ${ }^{59,60}$. The $K_{\mathrm{D}}$ values for dimerization of wildtype YibK and V139R YibK are $<1 \mathrm{nM}$ and $360 \mu \mathrm{M}$, respectively ${ }^{60}$. The introduction of $\mathrm{V} 139 \mathrm{R}$ resulted in $>8$-fold

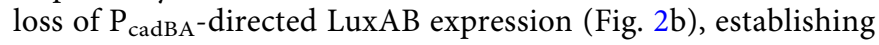


Fig. 1 Periplasmic PACE (pPACE) selection system. a Overview of PACE. Selection phage (SP, blue) encode an evolving protein (red) in place of the native phage gene III ( $\mathrm{gIII}$ ), which encodes essential phage protein plll. Host cells (tan) are transformed with a mutagenesis plasmid (MP, green) and one or more accessory plasmids (AP, purple) encoding selection-specific genes. The selection links the desired function of the evolving protein to expression of gene III. Induction of the MP with arabinose rapidly mutates the evolving gene. Phage encoding functional variants of the evolving protein trigger gIII transcription and pIII translation, and are thus able to propagate in a fixed-volume "lagoon", while phage with nonfunctional variants are diluted out of the lagoon over time. $\mathbf{b}$ Native E. coli CadC signaling function ${ }^{54,56}$. The CadC sensory domain (green) dimerizes under conditions of high $\mathrm{pH}$ and low lysine in the periplasm, leading to dimerization of the cytoplasmic component of CadC and activation of $\mathrm{P}_{\text {cadBA }} 54,56$. $\mathbf{c}$ Periplasmic PACE schematic. Phage encodes an evolving protein fused to a GCN4 leucine zipper. Following periplasmic export, GCN4 directs dimerization of the scFv-GCN4 species. Upon binding the target antigen (yellow), the dimeric evolving protein brings together two monomers of CadC linked to the antigen. Once in close proximity, the cytoplasmic DNA-binding domains of dimeric CadC cooperatively bind the DNA elements Cad1 and Cad2 of promoter $\mathrm{P}_{\text {cadBA, }}$ inducing transcription of $\mathrm{glll}$ and phage propagation.

that protein-protein affinity determines the degree of transcriptional activation at $\mathrm{P}_{\text {cadBA }}$.

To link binding in the periplasm to phage propagation, we drove gIII expression with $\mathrm{P}_{\text {cadBA }}$. We challenged phage encoding SS-YibK-SH2 to propagate in overnight culture on host cells expressing CadC-HA4 and $\mathrm{P}_{\text {cadBA-driven gIII. Phage encoding }}$ wild-type YibK propagated more than three orders of magnitude more efficiently in this periplasmic PACE system than V139R YibK phage, demonstrating that pPACE links target protein binding in the periplasm to phage propagation through $\mathrm{P}_{\text {cadBA }}$ activation and production of PIII (Fig. 2c).

Periplasmic phage-assisted evolution of YibK. To validate the pPACE selection for periplasmic protein-protein binding, we challenged the system to evolve homodimeric YibK variants starting from phage encoding the monomeric V139R variant. We adapted pPACE into the format of PANCE (phage-assisted noncontinuous evolution) $)^{37,38,41,61}$, a non-continuous form of PACE in which host-phage populations undergo serial daily passaging in lieu of continuous flow, permitting a less stringent and more sensitive initial selection. After three PANCE passages, phage titers increased robustly (Supplementary Fig. 3b).

YibK variants evolved mutations that restore YibK dimerization. On the YibK dimer interface, V139 forms a hydrophobic contact with A138' of its binding partner ${ }^{62}$. Evolving phage did not directly revert the V139R point mutation. However, in PANCE-evolved clone 3.7, residue A138 mutated to an aspartic acid (GCC to GAT), completely restoring affinity as measured by $\mathrm{P}_{\text {cadBA }}$ transcriptional activation (Fig. 2d, f). R146, which is in close proximity to R146', was converted to a cysteine residue in seven of eight sequenced phage (CGT to TGT; Fig. 2g; Supplementary Fig. 3d), resulting in stronger transcriptional activation of $\mathrm{P}_{\text {cadBA }}$ than wild-type YibK. Remarkably, we found that R146C results in an intermolecular disulfide bridge. The covalently bound species can be seen by SDSPAGE in purified YibK protein, as a $\sim 43 \mathrm{kDa}$ band representing the dimeric form of the $21.6 \mathrm{kDa}$ monomer (Fig. 2e, Supplementary Fig. $4 \mathrm{~b}, \mathrm{c}$ ). In whole-cell lysates, a $\sim 60 \mathrm{kDa}$ band representing the dimer of $30 \mathrm{kDa}$ YibK-SH2 can also be visualized (Supplementary Fig. 4d-e). Both dimeric species are lost upon the addition of reducing agent. Together, these results establish that the periplasmic selection platform is capable of restoring and improving the stable homodimerization of a monomeric protein by multiple mechanisms, including the evolution of novel disulfide bridges.

Periplasmic evolution of antibody-antigen affinity. Next, we applied pPACE to antibody-antigen binding. Full-length antibodies can be engineered into smaller forms such as single-chain variable fragments (scFvs), comprising only the heavy and light chain variable regions $\left(V_{H}\right.$ and $\left.V_{L}\right)$ tethered by a flexible synthetic linker ${ }^{1,2}$. ScFvs are small $(\sim 30 \mathrm{kDa})$, can be produced in E. coli, exhibit improved tissue penetration, and can be readily conjugated to drug molecules, effector proteins and chimeric antigen receptors ${ }^{1,3}$, making them prime candidate molecules for directed evolution approaches. Heterologous expression of scFvs in E. coli typically involves export into the periplasm using an $\mathrm{N}$-terminal signal sequence ${ }^{3,63}$.

We challenged pPACE to evolve scFv antibodies. We chose the $\Omega$-graft antibody $\mathrm{scFv}$, which targets the leucine zipper GCN4 with $K_{\mathrm{d}} \sim 500 \mathrm{pM}^{39,64}$. To determine whether an antibody-antigen interaction could drive CadC dimerization, we expressed CadC-HA4 and $\Omega$-graft-SH2, with or without coexpression of a monomeric form of the leucine zipper GCN4 ${ }^{65}$ (GCN4(7P14P)) fused to SH2. In this architecture, the binding of $\Omega$-graft to GCN4 drives dimerization of CadC-HA4 bound to $\Omega$-graft-SH2 and a CadC-HA4 molecule bound to GCN4-SH2, creating a four-part complex (Fig. 3a). The addition of GCN4 led to a 30-fold increase in $\mathrm{P}_{\text {cadBA-driven }}$ LuxAB expression (Fig. 3b). In contrast, the substitution of a $\Omega$-graft double point mutant L231F F232A, which impairs binding to GCN4 by $>7,000$-fold ${ }^{64}$, in place of wild-type $\Omega$-graft led to a 55 -fold decrease in transcriptional activation (Fig. 5b). Collectively, these results indicate that our selection can link scFv target binding to transcriptional activation of $\mathrm{P}_{\text {cadBA }}$.

To determine whether pPACE can distinguish between functional and nonfunctional forms of $\Omega$-graft, we performed a competitive mock-selection experiment without mutagenesis. We seeded host cells expressing CadC-HA4 and GCN4-SH2 and encoding $\mathrm{P}_{\text {cadC }}$-driven gene III on the AP with a mixture of selection phage containing a 1:1,000 ratio of unmutated $\Omega$ graft-SH2 selection phage to L231F F232A mutant-SH2 selection phage and carried out PACE and PANCE. Within $12 \mathrm{~h}$ of PACE or following two PANCE passages, unmutated $\Omega$-graft variants dominated both populations, enriching $\geq 1000$-fold (Supplementary Fig. 5a), demonstrating that the selection platform can be used to selectively propagate phage encoding a target-binding antibody scFv.

Regulating scFv periplasmic export. In the small volume of the periplasm, minor changes in protein expression level have a large impact. An evolving SP might achieve increased fitness by modifying the promoter driving scFv expression to increase scFv levels and compensate for a poor $K_{\mathrm{D}}$. We reasoned that controlling scFv export to the periplasm would be desirable to maintain selection pressure. Further, regulating the level of periplasm-targeted $\mathrm{scFv}$ protein could drive two simultaneous selections: for high affinity to the target and for increased solubility of the $\mathrm{scFv}$, to raise the effective concentration of $\mathrm{scFv}$. We therefore adapted a key aspect of a related PACE selection, soluble expression PACE or SE$\mathrm{PACE}^{39}$. SE-PACE uses a trans-splicing intein to reconstitute two fragments into a single functional protein, integrating transcription from two promoters into one output ${ }^{39,66}$. In SE-PACE, inteinmediated splicing reconstitutes the signal sequence peptide of pIII, which must enter the periplasmic space for phage to propagate ${ }^{39}$. 

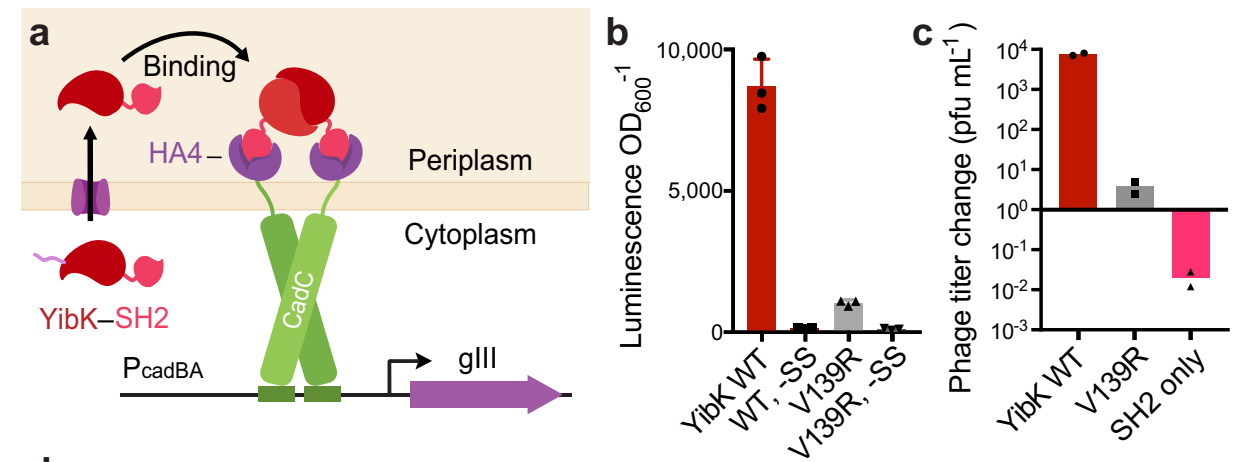

\begin{tabular}{cccccc}
\multicolumn{1}{l}{ d } & \multicolumn{5}{l}{ Residue number } \\
\hline YibK variant & 138 & 139 & 144 & 146 \\
\hline WT & A & V & A & R \\
V139R & A & R & A & R \\
3.6 & A & R & D & C \\
3.7 & D & R & D & C
\end{tabular}

e
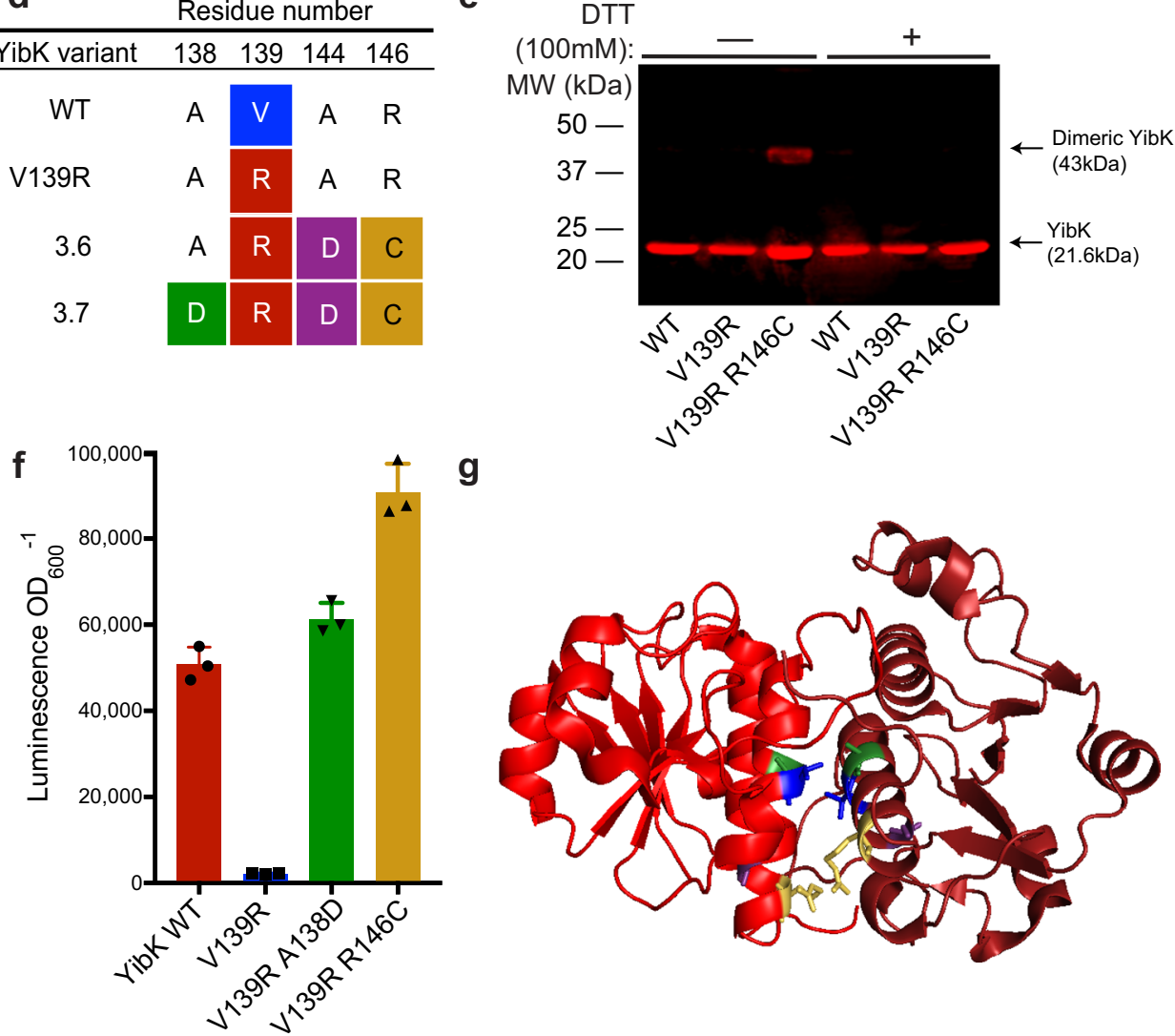

g

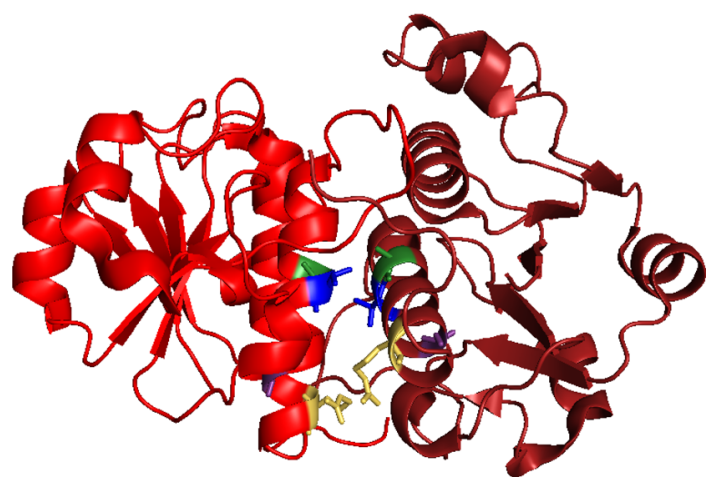

Fig. 2 Periplasmic phage-assisted non-continuous evolution of the dimeric knottin YibK rescues binding mutants and evolves new disulfide bonds. a Schematic of homodimeric YibK selection. HA4 monobody (purple) recruits SH2 (pink) to CadC, and CadC monomers (green) are brought together by homodimerization of YibK (red). b Transcriptional activation assay comparing the performance of wild-type YibK-SH2 construct (WT) to the V139R binding mutant in the presence and absence of a signal sequence (SS) to direct periplasmic export. The architecture of the luciferase-based transcriptional reporter is shown in Supplementary Fig. 2a. Bar values and error bars represent the mean and s.d. of three independent biological replicates. c Phage propagation assay. Mid-log-phase cultures of selection strains were inoculated with phage and allowed to propagate overnight before determining titer. WT SS-YibK-SH2 phage enriches robustly, while the YibK V139R point mutant in the same construct enriches weakly and phage encoding only SP-SH2 fail to enrich. Bar values represent the mean of two independent biological replicate experiments carried out on separate days. (d) PANCE of YibK variant V139R evolves variants 3.6 and 3.7, showing two compensatory point mutations, A138D and R146C. R146C establishes a novel intermolecular disulfide bridge, resulting in a covalently bonded dimeric species that can be eliminated by the addition of a reducing agent, as shown by Western blot of purified YibK protein (e). The full gel image and corresponding Coomassie gel are provided in Supplementary Fig. 4b-c. The experiment was repeated once with similar results. $\mathbf{f}, \mathbf{g}$ A138D restores wild-type activity in a V139R background in transcription assays (f), and likely forms a salt bridge with R139, as seen in the crystal structure of YibK dimer (g). Positions 138 (green) and 139 (blue) are in contact at the dimer interface. PDB ID $=1 \mathrm{MXI}{ }^{62}$. Bar values and error bars in (f) represent the mean and s.d. of three biological replicates. Source data are provided as a Source Data file.

We split the PhoA-derived signal sequence (SS) ${ }^{58}$ into two halves, consisting of amino acids $1-8$ and 9-21 (Supplementary Fig. 6). These two halves were fused, respectively, to the $\mathrm{N}$ - and C-terminal portions of the Nostoc punctiforme ( $\mathrm{Npu}$ ) transsplicing DnaE intein ${ }^{67}$. SS amino acids 1-8 were fused to the $\mathrm{N}$-terminal half of the Npu intein on a host AP1, ensuring that phages are not able to evolve the increased expression of this component. The C-terminal half of the Npu intein, fused to SS amino acids 9-20 and the evolving scFv, was encoded on the selection phage. Following translation of both fusion proteins, intein-mediated splicing reconstitute full-length SS-scFv, allowing periplasmic export (Supplementary Fig. 6). 

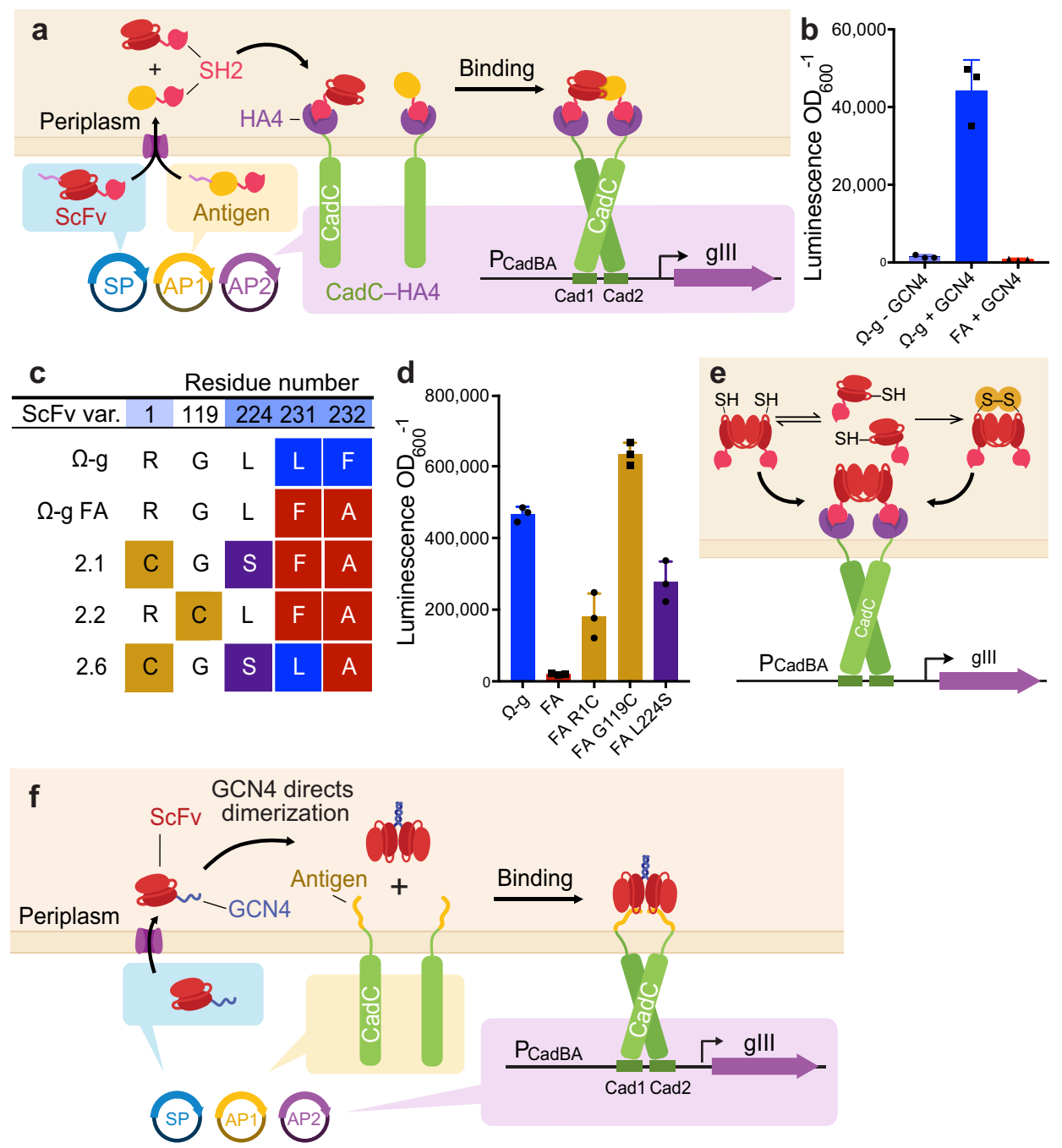

Fig. 3 Initial design of pPACE and mechanism of selection survival through homodimerization. a Schematic overview of initial selection design. b Luminescence-based transcriptional activation assay comparing the performance of $\Omega$-graft (abbreviated $\Omega$-g) to the L231F F232A (here abbreviated FA) binding mutant in the presence and absence of its cognate antigen, a monomeric form of GCN4(7P14P) (abbreviated GCN4 in b) in the system diagrammed in (a). c PACE generates multiple variants with spontaneous N-terminal or 4X GGGS linker cysteine residues in addition to variants reversing mutation L231F. Full results can be found in Supplementary Fig. 5b. d Transcriptional activation assay. In a non-binding background, N-terminal cysteines drive partial or complete restoration of $\mathrm{P}_{\text {cadBA }}$ transcriptional activation, suggesting a mechanism of surviving the selection by the formation of novel disulfide bonds that generate covalent homodimeric scFvs, as shown in (e). Homodimeric scFv-SH2 fusions are able to bind drive CadC-HA4 dimerization without the involvement of the antigen. Bar values and error bars in (b) and (d) represent the mean and s.d. of three independent biological replicates. (f) Novel selection architecture designed to alleviate dimerization issues addressed above. A signal sequence (not depicted) directs export of scFv-GCN4 to the periplasmic space. The dimerizing leucine zipper GCN4 can be used to direct dimerization of scFvs in a predictable manner. Source data are provided as a Source Data file.

Using the $\Omega$-graft pPACE selection described above, we observed that expressing $\Omega$-graft-SH2 with its SS split into two polypeptides, each fused to half of the $\mathrm{Npu}$ intein, led to robust phage propagation, indicating reconstitution of the SS. In contrast, when we omitted the C-terminal domain of $\mathrm{Npu}$ from the $\mathrm{SS}_{9-20^{-}} \Omega$ graft-SH2 construct, phage failed to propagate (Supplementary Fig. 6c). Similarly, the expression of the $\mathrm{SS}_{1-8}-\mathrm{NpuN}$ component is necessary for propagation (Supplementary Fig. 6d). We further found that by expressing $\mathrm{SS}_{1-8}-\mathrm{NpuN}$ under small-molecule induction in the presence of $\mathrm{NpuC}-\mathrm{SS}_{9-20}-\Omega$-graft $(34.8 \mathrm{kDa})$, we could drive periplasmic expression of $\Omega$-graft $s c F v(30.2 \mathrm{kDa})$ in a dose-dependent manner (Supplementary Fig. $6 \mathrm{~g}, \mathrm{~h}$ ). This demonstrates that Npu can regulate the reconstitution of full-length SS for periplasmic export of scFvs and $\mathrm{P}_{\mathrm{cadBA}}$ activation.

Under this intein-regulated system, the total amount of $s c F v$ exported to the periplasm, and thus available direct CadC dimerization, is limited by the availability of the intein-SS fragment encoded on the host AP. The researcher can modify the expression level of intein-SS ${ }_{1-8}$ fragment to limit the reconstitution of SS-scFv, and thus the amount of $s c F v$ exported to the periplasm, creating selection pressure for efficient expression of soluble scFv.

Evolution of $\Omega$-graft and overcoming scFv homodimerization. Next, we challenged pPACE to correct the L231F F232A binding mutation in the $\Omega$-graft antibody scFv, using both a full-length $\mathrm{N}$ terminal SS sequence and the intein-SS strategy described above, in order to select for affinity alone or affinity and soluble periplasmic expression. We aimed to apply pPACE to restore binding to GCN4 by correcting mutation L231F F232A.

PACE experiments using our original selection architecture (Fig. 3a) resulted in two genotypic outcomes. First, close to half of 
phage reverted mutation $\mathrm{L} 231 \mathrm{~F}$ to the wild type within $96 \mathrm{~h}$ of pPACE. Second, scFv variants developed cysteine residues at their $\mathrm{N}$-termini or within the 4X GGGS linker connecting scFv VH and VL domains (Fig. 3c). Linker cysteines in particular appeared mutually exclusive to the desired L231F reversion (Supplementary Fig. 5b). We found that at both positions, a cysteine substitution resulted in higher transcriptional activation than reversion of position 231 to Leu (Fig. 3d). The insertion of a C-terminal Cys residue has been used to manufacture stable dimeric scFvs through the formation of a covalent disulfide ${ }^{68}$. We reasoned that an $\mathrm{N}$-terminal or linker Cys residue might form a similar covalent linkage, generating stably homodimeric scFv-SH2. This homodimer circumvents the target-binding selection by binding two CadC-HA4 molecules and bring them into close proximity, without the involvement of the antigen (Fig. 3e).

To prevent circumvention of the target-binding selection, we modified the selection architecture by fusing the GCN4(7P14P) antigen directly to $\mathrm{CadC}$ in place of $\mathrm{HA} 4$, to eliminate the possibility of $\mathrm{scFv}$ homodimerization resulting in selection survival (Fig. 3f). We created obligate homodimeric scFvs by removing the now-redundant $\mathrm{SH} 2$ domain fusion and either preinstalling an $\mathrm{N}$-terminal cysteine in the $\Omega$-graft scFv (Fig. $4 \mathrm{a}$ ), or, as a more general strategy, by fusing a homodimerizing GCN4 leucine zipper domain C-terminal to the scFv (Fig. 3f; Fig. 5a, Supplementary Fig. 7). This strategy ensures that efficient dimerization does not depend on the properties of the $s c F v$ being evolved, since different scFvs homodimerize at different rates $^{69}$. In this second-generation selection architecture, a dimeric $s c F v$ antibody must bind two CadC-fused antigens to activate

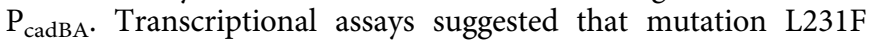
accounts for the loss of binding, and that F232A alone has little effect (Supplementary Fig. 8e). We therefore considered reversion of F232A to be unnecessary in desired selection outcomes.

Using this second-generation architecture, phage encoding $\Omega$ graft showed three orders of magnitude higher levels of propagation in overnight enrichment assays than phage encoding $\Omega$-graft L $231 \mathrm{~F}$ (Fig. 4b, c). Incorporation of a nonsense mutation at position 100 (W100*) also led to strong de-enrichment of phage (Fig. 4b).

We challenged pPACE using the second-generation architecture to correct a stop codon at W100 in addition to the L231F binding defect mutation. Within $96 \mathrm{~h}$ of pPACE, phage reverted mutations correcting both deleterious mutations in population 1 (Fig. 4e, Supplementary Fig. 9a). In population 2, we used the split-intein signal sequence strategy described above to regulate periplasmic scFv expression in host cells (Fig. 4a, f). Due to the decreased fitness of intein-SS phage compared to phage with full-length SS (Fig. 4b), we did not challenge population 2 to correct a stop codon. Mutation F231L was present in $\sim 50 \%$ of this population by $96 \mathrm{~h}$ and dominated the population by $156 \mathrm{~h}$ (Supplementary Fig. 9b). Phage in different populations accessed leucine codons at position 231 via two distinct point mutations, converting TTC to TTA or CTC (Fig. 4e, f). Importantly, we observed no new cysteines arising during evolution (Fig. 4g; Supplementary Fig. 9a). These results suggest that the second-generation pPACE selection prevents phage from passing the selection by evolving stable $\mathrm{scFv}-\mathrm{SH} 2$ homodimers alone, and requires a tight scFv-antigen interaction in order to activate $\mathrm{P}_{\text {cadBA }}$.

In population 2 , which used the intein-SS strategy, two point mutations, F231L and L224S, enriched as separate solutions present at a similar frequency at $96 \mathrm{~h}$. Mutations F231L and L224S were observed in the same variant by $112 \mathrm{~h}$ (Supplementary Fig. 9b). Mutation L224V was previously reported to enhance cytoplasmic solubility of the $\Omega$-graft $\mathrm{scFv}^{39}$. We compared the soluble expression of $\Omega$-graft $\mathrm{scFv}$ and variant L224S with an N-terminal PhoA SS from the promoter $\mathrm{P}_{\mathrm{T} 7 \mathrm{Lac}}$ in $\mathrm{BL} 21^{*} \mathrm{DE} 3$ cells by western blotting.
We found that L224S increased soluble expression of $\Omega$-graft by roughly 8 -fold (Supplementary Fig. 9c, d).

Together, these findings demonstrate that pPACE can restore affinity of an antibody to an antigen, and that regulating periplasmic export of the evolving species using a split-intein signal sequence can support the evolution of improved soluble expression and improved binding. These results also show that the second-generation pPACE system avoids outcomes that circumvent the selection by homodimerizing the evolving protein, rather than by binding the target.

Periplasmic PACE of novel trastuzumab scFv variants. We used the second-generation pPACE selection to evolve an $\mathrm{scFv}$ form of the antibody trastuzumab to bind a new target antigen. Trastuzumab targets the oncogenic receptor Her2 and is a successful first-line treatment for Her $2^{+}$breast cancers. Most trastuzumabresponsive tumors develop resistance to the drug within one year ${ }^{70}$. Second-line treatments can overcome resistance using multi-specific engineered antibodies, which combine variable domains of two or more mAbs to simultaneously target several epitopes, such as Her3, EGFR and VEGF kinase receptors ${ }^{71-73}$. The ability of pPACE to rapidly evolve affinity to novel epitopes could further broaden the targeting capacity of engineered multispecific antibodies.

The Her2 mimetic peptide H98 was identified in a peptide library screen for trastuzumab binding, and bears structural similarity but no sequence homology to Her $2^{74}$. We sought to apply pPACE to evolve an scFv form of trastuzumab with a higher affinity for the $\mathrm{H} 98$ peptide. We evolved trastuzumab $\mathrm{scFv}$ in the second-generation pPACE selection using either full-length SS or the split-intein SS strategy, resulting in mutually exclusive outcomes within $96 \mathrm{~h}$ of evolution. The H98 antigen was presented as a CadC-H98 fusion driven by a weak constitutive promoter on the AP. Trastuzumab was expressed as an scFv-GCN4 fusion to ensure dimerization, as we found that the use of a larger domain such as YibK to direct dimerization resulted in poor phage propagation (Fig. 5b), possibly due to excessive crowding of the periplasmic space.

Phages were allowed a 24-h period of evolutionary drift when pIII was provided freely with elevated mutagenesis ${ }^{30}$, to generate a large and diverse phage library. Phages were then subjected to high-stringency pPACE at increasing flow rates until titers plateaued (Fig. 5c, Supplementary Fig. 10). In populations 1 and 2 , phage encoded the full-length SS. Both populations converged on a single point mutation, H91Y (variant 1.1, Fig. 5d). In population 3, periplasmic export was restricted through the split-intein strategy described above, leading to enrichment of a single variant (3.2) with mutations A34D Y49S. Periplasmic PACE experiments carried out at further increased stringencies did not result in the enrichment of any additional point mutations in the scFv (Supplementary Fig. 11).

Computational modeling indicates that trastuzumab interacts with H98 through heavy chain residues V33, R50, and Y105, and light chain residues $\mathrm{T} 94$ and $\mathrm{N} 30^{74}$. In the trastuzumab crystal structure, residue T94 is proximal to residue H91 (H91Y in variant 1.1), and residue $\mathrm{N} 30$ is proximal to residue A34 (A34D in 3.2) (Supplementary Fig. 10e). Light chain residue Y49 is adjacent to residue $\mathrm{A} 34$ in a $\beta$-sheet, and mutation Y49S (variant 3.2) may help to accommodate the substitution of alanine for a bulky, charged aspartic acid at position 34 (PDB ID: $1 \mathrm{~N} 8 \mathrm{Z}^{75}$ ).

Trastuzumab and evolved variants show a similar, characteristic change in mobility consistent with the reduction of disulfides during SDS-PAGE under reducing conditions, suggesting that intra-chain disulfides are retained in evolved variants (Supplementary Fig. 12a). To examine the role of intra-chain disulfides in 
a
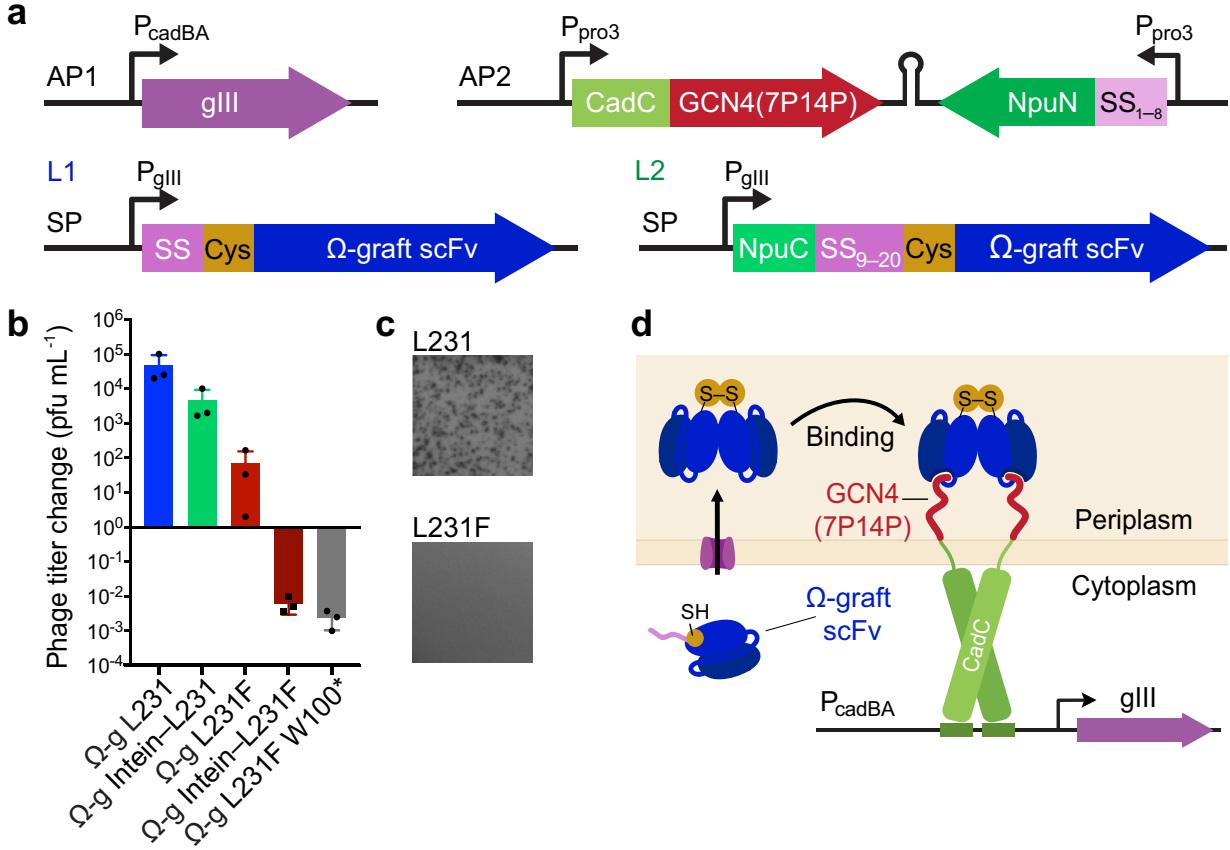

d

e

L1: SS- $\Omega$-graft scFv L231F W100*

$\mathbf{f}$
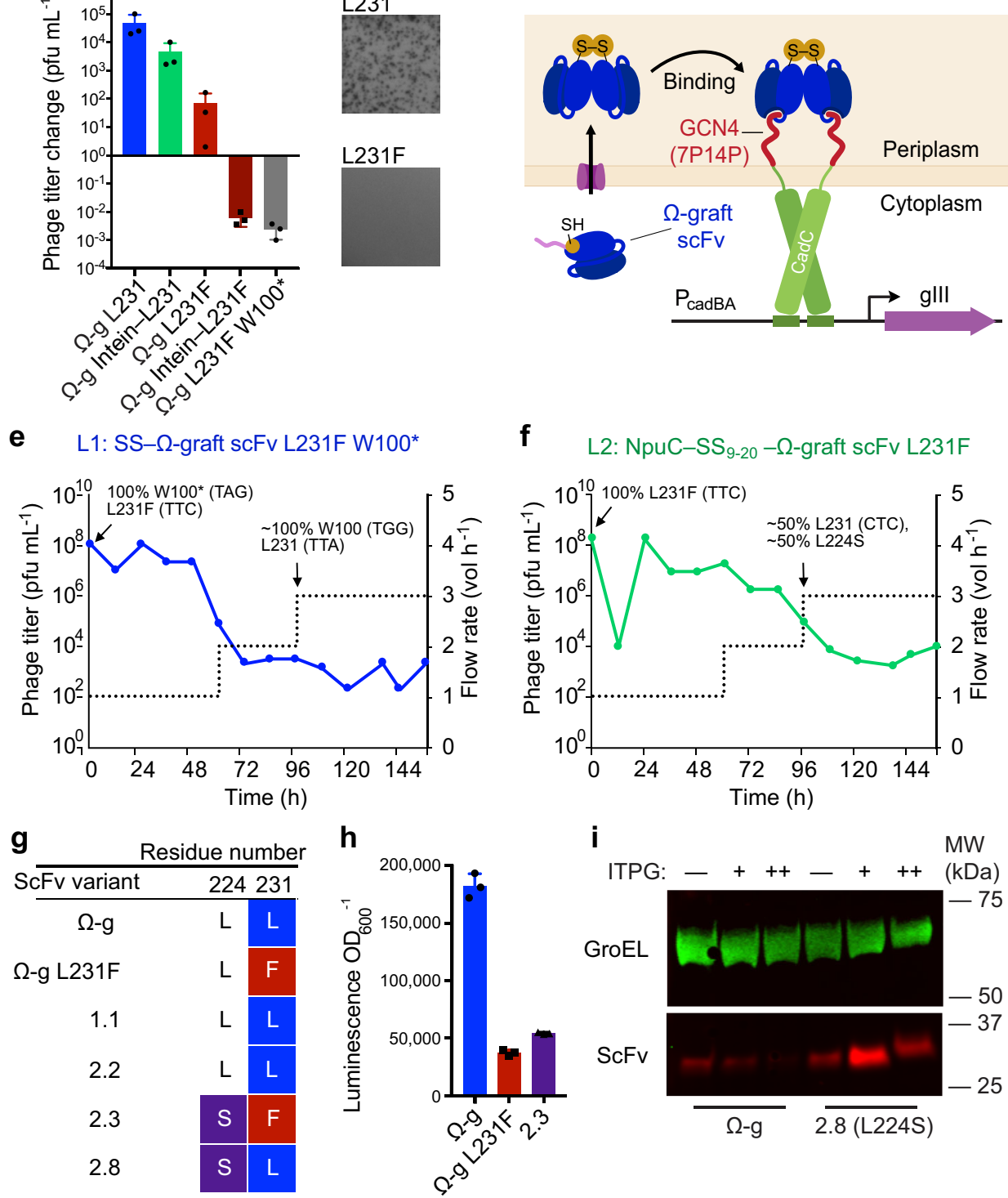

Fig. 4 Second-generation pPACE selection reverts a binding mutant in $\mathbf{\Omega}$-graft scFv. a Schematic of periplasmic PACE architecture 2 components. $\Omega$-graft $(\Omega-g)$ scFvs form covalent dimers through N-terminal cysteine residues. GCN4 monomeric variant 7P14P is used to avoid background dimerization of CadC. Promoter $\mathrm{P}_{\text {pro3 }}$ is a low-level constitutive promoter ${ }^{39}$. $\mathrm{P}_{\mathrm{g} \text { III }}$ is a native phage promoter. $\mathbf{b}$ Overnight phage propagation assay of $\Omega$-graft scFv variant $\mathrm{SP}$, illustrating the effect of L231F on phage propagation. Introduction of a stop codon into position 100 of scFv (L231F-STOP) prevents phage propagation. Splitting the signal sequence using an intein (intein-L231/F) leads to reduced propagation. Bar values and error bars represent mean and s.d. of three biological replicates conducted on separate days. c Plaque assay visualizing overnight expansion of intein-SS $9-20$ phage variants L231 and L231F as in (b). Full plates provided in Supplementary Fig. $8 \mathrm{c}, \mathrm{d}$. $\mathbf{d} \Omega$-graft selection overview. After periplasmic export and SS cleavage, a cysteine is exposed at the $\mathrm{N}$-terminus to mediate covalent disulfide bonding of two scFv monomers as described in Fig. 3e. Binding of GCN4 by dimeric scFvs leads to activation of $P_{\text {cadBA. }}$ e, $\mathbf{f}$ PACE was carried out over $156 \mathrm{~h}$ using full-length SS-scFv phage (e) or split-intein SS-scFv phage (f). To impose additional challenges, full-length SS-scFv phage were also challenged to correct a nonsense mutation. By 96 h, phage had converged upon solutions shown in (g) and in Supplementary Fig. $9 a$, b. Duplicates of each PACE experiment were evolved with similar outcomes, correcting W100* and enriching F231L in the replicate of (e) and discovering L224S and F231L in replicate of (f). $\mathbf{h}$ Luminescence assay shows increased $\mathrm{P}_{\text {cadBA }}$ activation as a result of L224S in an L231F background. Bar values and error bars represent mean and s.d. of three biological replicates. i Western blot showing $\Omega$-graft and L224S evolved mutant, expressed from $\mathrm{P}_{\text {T7Lac }}$ in BL21*DE3 cells. L224S increases soluble $\Omega$-graft scFv expression by roughly 8 -fold. This experiment was repeated in biological triplicate on separate days with similar results. Full gel and densitometry analyses are provided in Supplementary Figs. 9c, $d$ and $15 \mathrm{~g}$ and in the Source Data file. 
a
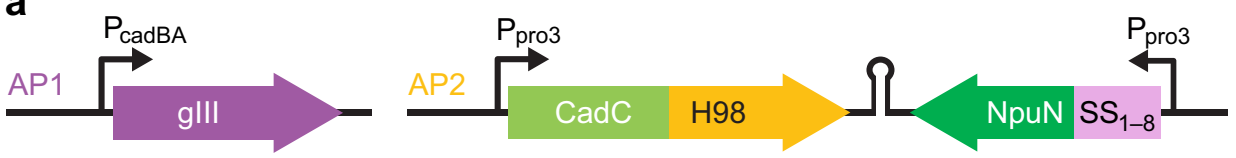

L1, L
SP
$\mathbf{b}=$

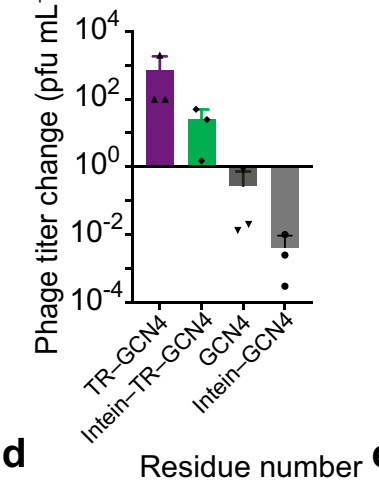

C Trastuzumab scFv PACE

\begin{tabular}{ll|l|l} 
d & \multicolumn{4}{c}{ Residue number } \\
\hline ScFv var. & 34 & 49 & 91 \\
\hline TR & A & Y & H \\
1.1 & A & Y & Y \\
2.1 & A & Y & Y \\
3.2 & D & S & H
\end{tabular}

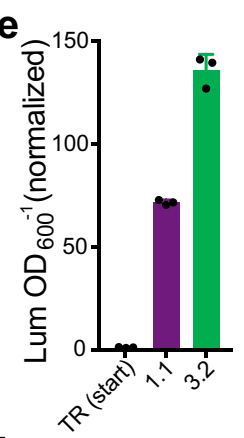

f
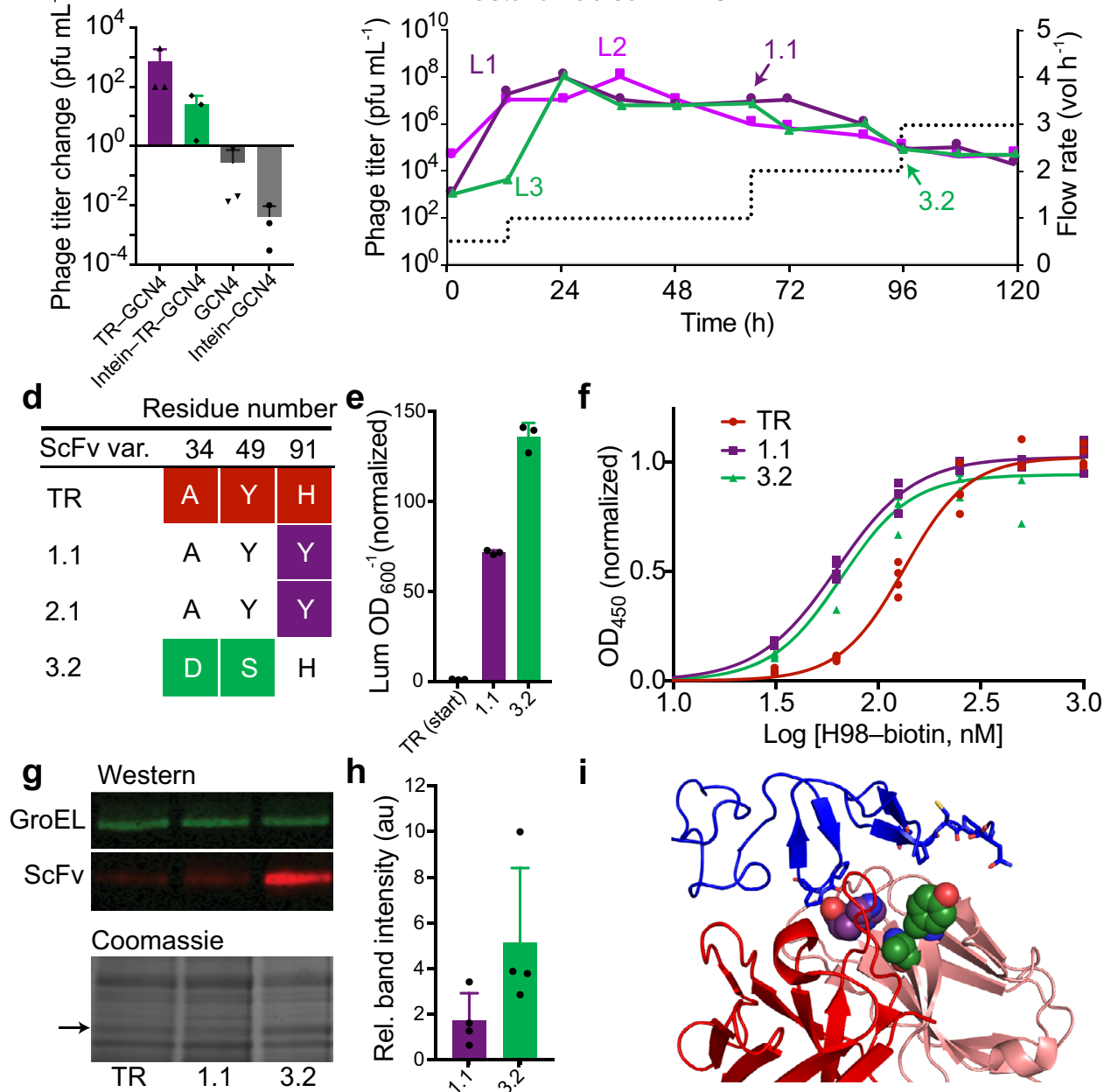

i

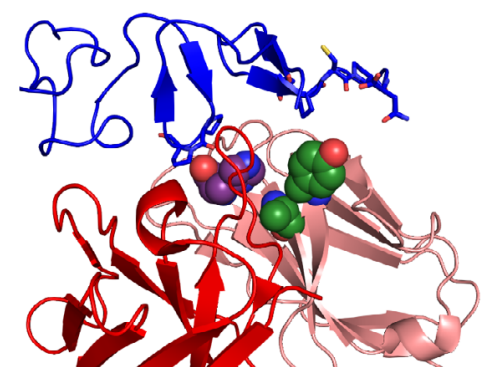

Fig. 5 Evolution of trastuzumab variants with improved binding to a Her2-mimetic peptide. a Components of the second-generation periplasmic PACE system to evolve trastuzumab. The H98 peptide is a structural homologue of the Her2 epitope. A C-terminal dimeric GCN4 peptide directs dimerization of scFvs. b Phage propagation assay of starting genotypes and negative controls. Sequences with intein-split SS are indicated as 'intein'. c PACE was carried out over $120 \mathrm{~h}$ using full-length (lagoons L1-L2, purple) or split-intein signal sequence (lagoon L3, green). By 96 h, all three lagoons converged on discrete solutions, shown in (d) and in Supplementary Fig. 10a. e Luminescence assay with trastuzumab (abbreviated TR, red) and evolved trastuzumab variants demonstrates increased $\mathrm{P}_{\text {cadBA }}$ activation. Luminescence/ $\mathrm{OD}_{600}$ values are shown relative to that of trastuzumab. $\mathbf{f}$ ELISA shows modest improvement in binding. Values represent the mean and individual data points of four technical replicates from the same protein preparation. Data points at far ends of the binding curve, used to verify top and bottom values, can be found in Supplementary Fig. 10c. This experiment was repeated with four separate protein preparations and gave similar results. Average $\mathrm{EC}_{50}$ and Hill slope values from all replicate experiments can be found in Table 1. PAGE analysis of purified protein used in this representative ELISA is shown in Supplementary Fig. 16b. $\mathbf{g}, \mathbf{h}$ Western blot and Coomassie-stained gels of TR and evolved variants expressed from the T7Lac promoter in BL21*DE3 cells, showing improved soluble expression of variant 3.2. Full gels are shown in Supplementary Fig. 15a, b. Densitometry data reflects mean and s.d. of Western blot method and includes four independent biological replicates conducted on separate days. $\mathbf{i}$ Location of individual evolved mutations from PACE in the crystal structure of trastuzumab Fab bound to Her2 (PDB ID: $1 \mathrm{~N} 8 \mathrm{Z}^{75}$ ). Mutations are colored by lagoon origin as in (c). Bar values and error bars in (b), (e), and (h) represent the mean and s.d. of three independent biological replicates. Source data are provided as a Source Data file.

the stability and binding of trastuzumab variants, we abrogated the possibility of disulfide formation by replacing the disulfideforming cysteine residues with serines. In the absence of disulfide bonds, both trastuzumab and evolved variants failed to induce transcription from $\mathrm{P}_{\text {cadBA }}$ (Supplementary Fig. 12b). These results further suggest that trastuzumab binding is likely dependent on intra-chain disulfides, in agreement with the findings of Wörn and Plückthun that expression of trastuzumab scFv without disulfide bonds results in insoluble $\operatorname{protein}^{76}$, and that these disulfides are preserved through pPACE. To ensure that the 
Table 1 Properties of trastuzumab scFv and evolved variants.

\begin{tabular}{lccl} 
& $\mathbf{E C}_{\mathbf{5 0}}(\mathbf{n M})^{\mathbf{a}}$ & Hill slope $^{\mathbf{a}}$ & $\mathbf{T}_{\mathbf{M}}\left(\mathbf{C}^{\circ}\right)$ \\
\hline TR & $160.7 \pm 26.2$ & $3.1 \pm 0.6$ & 68.5 \\
1.1 & $63.6 \pm 3.0$ & $2.6 \pm 1.4$ & 72.5 \\
3.2 & $77.6 \pm 15.0$ & $3.0 \pm 1.0$ & 62.5
\end{tabular}

Trastuzumab is abbreviated as TR. Values were determined by pooling means from four ELISA experiments conducted with separate protein preps, each with four technical replicates per ELISA experiment, and calculating mean and s.d. of pooled means. Melting temperature data reflects mean of two experiments conducted with separate protein preps, each consisting of four technical replicates. Source data are provided as a Source Data file.

accumulation of insoluble cytoplasmic scFv does not impair host cell fitness, we carried out a growth timecourse, and found that scFv expression, with or without split-intein SS, had little to no effect on host cell growth (Supplementary Fig. 13).

Evolved variant 1.1 showed $\sim 2.5$-fold improved binding to H98 as measured by ELISA and little change in soluble expression (Fig. 5f-h, Table 1, Supplementary Fig. 10c, d). Evolved variant 3.2 was selected using the split-intein SS selection and showed a $\sim 2$-fold increase in affinity (Fig. 5f-h, Table 1, Supplementary Fig. 10c, d). To support ELISA data, we also carried out microscale thermophoresis (MST) (Supplementary Fig. 14, Supplementary Table 5). We note that in MST experiments, the upper bound of the binding curve was not accessible due to solubility limits of the H98 peptide in aqueous buffer (Supplementary Fig. 14), which may affect the accuracy of $\mathrm{K}_{\mathrm{d}}$ determination. However, $\mathrm{EC}_{50}$ values determined by MST and ELISA reflect similar fold improvements for evolved variants over the starting $s c F v$.

Variant 3.2 showed substantial increases in soluble periplasmic expression ( $~ 5$-fold as measured by western blotting and 2.5 -fold as measured by Coomassie staining; see Supplementary Fig. 15), suggesting that restricting the level of $\mathrm{scFv}$ export to the periplasm selected for enhanced solubility. Evolved variants showed unchanged binding to Her2 in ELISA compared to that of trastuzumab scFv (Supplementary Fig. 10b). Evolved variants showed relatively unchanged thermal stability. Unevolved trastuzumab scFv had a melting temperature of $68.5^{\circ} \mathrm{C}$. We observed $\mathrm{T}_{M}$ increase of $+4.0^{\circ}$ for variant 1.1 and a $\mathrm{T}_{M}$ decrease of $-5^{\circ} \mathrm{C}$ for variant 3.2 (Supplementary Fig. 15 f, Table 1 ).

\section{Discussion}

Continuous directed evolution has the potential to significantly streamline the development of antibodies, but disulfidecontaining proteins represent a significant challenge for current continuous evolution methods, which occur in the reducing cytoplasmic space. We developed a method for the continuous evolution of protein binding that takes place in the bacterial periplasm. Periplasmic PACE can rapidly generate proteins with improved binding and expression properties from a starting gene within several days of evolution. While the packaging limit of the M13 bacteriophage $(12 \mathrm{~kb})$ likely makes the evolution of full-length immunoglobulin (IgG) antibodies unwieldy, pPACE focuses evolution to the domains containing the complement-determining regions, maximizing the likelihood of discovering mutations that improve binding without increasing immunogenicity ${ }^{77}$. ScFv can then be grafted into scaffolds to produce full-length IgGs. Periplasmic PACE supports native disulfide bonds, which can be critical for the folding and stability of scFvs and other proteins in both prokaryotic and eukaryotic contexts $^{78}$. Splitting the signal sequence into two halves, with one half expressed at a controlled level on a host plasmid, allows the researcher to define the extent of export to the periplasm, further modulating selection stringency.

We applied pPACE to evolve YibK variants with restored binding via two novel mechanisms in only three serial passages, $\Omega$-graft antibody variants with restored binding and 8-fold improved solubility within $96 \mathrm{~h}$ of pPACE, and trastuzumab variants with up to 5-fold improved solubility and 2.5-fold improved binding affinity to a peptide antigen within $96 \mathrm{~h}$ of pPACE. Taken together, these studies establish that pPACE can evolve improved binding and expression profiles of antibodies and other proteins in the periplasmic space on short timescales.

In an oxidizing environment such as the extracellular space, intra-chain disulfides are highly conserved, and can make the $\Delta G$ of folding more favorable by $4-5 \mathrm{kcal} \mathrm{mol}^{-1}$, corresponding to an increase in folded states over unfolded states of roughly three orders of magnitude ${ }^{78}$. For non-intrabody applications such as CAR-T therapy, engineering disulfide-free scFvs is generally not desirable or necessary. Periplasmic PACE therefore offers a complementary strategy to other intracellular evolution methods by enabling continuous evolution for binding activity and soluble expression while conserving native disulfide linkages.

The properties of the periplasm offer opportunities that pPACE is well-suited to exploit. Protein channels in the outer membrane of $E$. coli render the periplasm permeable to water, ions, and hydrophilic solutes up to $\sim 600 \mathrm{Da}$ in size ${ }^{79}$. Further, the $\mathrm{pH}$ of the periplasm mirrors the $\mathrm{pH}$ of the extracellular environment ${ }^{80}$. The composition of the growth medium used in pPACE may strongly influence the folding and activity of evolving proteins. We speculate that pPACE might be used in the evolution of proteins with unusual $\mathrm{pH}$ requirements, and could be leveraged for applications involving small-molecule substrates.

Due to the innate constrains of E. coli expression and the architectural requirements of the CadC selection, periplasmic PACE is appropriate for the evolution of binding towards antigens that are small and well-behaved in the E. coli periplasm, do not contain essential glycosylations, and do not homodimerize. Large mammalian cell-surface antigens are not likely to behave well in pPACE, while peptides, very small proteins, and bacterial proteins are more likely to be compatible with pPACE. In this work, we have shown evolution towards peptide antigens (e.g, GCN4, $0.4 \mathrm{kDa})$ and YibK $(21.6 \mathrm{kDa})$. First-generation architecture is appropriate for use with monomeric evolving proteins, while second-generation pPACE is appropriate for dimeric evolving proteins and antigens that can tolerate an $\mathrm{N}$-terminal fusion.

ScFv phage with split-intein signal sequence propagated less robustly than their full-length SP counterparts (Figs. 4b, 5b). Under intein-regulated conditions only, both $\Omega$-graft and trastuzumab phage evolved improved soluble periplasmic expression. Limiting the rate of periplasmic export appears to impose selection pressure for both soluble periplasmic expression and affinity. In a regime limited by the availability of the $\mathrm{NpuN}-\mathrm{SS}_{1-8}$ fragment, mutations that improve overall expression levels are unlikely to have a large effect, as excess intein-SS ${ }_{9-20}-\mathrm{ScFv}$ construct simply accumulates in cytoplasm. Notably, we did not observe increased $\mathrm{scFv}$ in the insoluble pellet fraction following inteinregulated pPACE (Supplementary Figs. 9d and 15c, d). We speculate that splitting the signal sequence selects for variants that limit aggregation or degradation occurring after intein-mediated splicing, mediate rapid periplasmic export, or facilitate folding in the periplasm.

High-micromolar and low-nanomolar $K_{\mathrm{D}}$ variants of YibK and $\Omega$-graft performed very differently in pPACE. YibK variant 3.7 and $\Omega$-graft variant 2.8 evolved beneficial mutations in addition to the mutation expected to restore binding affinity to lownanomolar $K_{\mathrm{D}}$ levels. In the case of trastuzumab and the Her2 
mimetic peptide H98, however, only modest affinity improvements were evolved from an initially moderate $K_{\mathrm{D}}$ (the $K_{\mathrm{D}}$ of trastuzumab IgG-H98 interaction is reported to be $\left.1.4 \mu \mathrm{M}^{81}\right)$. This outcome may reflect the limited surface area of H98 available for molecular interaction, or may suggest stringency limitations in the selection. Consistent with these possibilities, further decreasing expression of antigen and pIII did not lead to enrichment of new trastuzumab genotypes (Supplementary Fig. 11). Indeed, mutations A34D and Y49S were not observed after high-stringency evolution, suggesting that expression increases may saturate the limited available H98 in this selection regime. We speculate that stringency might be further elevated by providing a competitive binder at the late stages of the selection.

We have shown that periplasmic PACE can improve both affinity and solubility of $\Omega$-graft and trastuzumab scFvs, and can generate variants of homodimeric YibK with non-covalent and covalent linkages between subunits. To our knowledge, periplasmic PACE represents the first PACE selection for function in a cell compartment other than the cytoplasm, and the first continuous selection in the periplasmic space. We anticipate this system may be particularly useful for rapid optimization of binding and solubility, especially when evolving antibodies to engage antigens that contain disulfide bonds and are incompatible with cytoplasmic PACE.

\section{Methods}

Nuclease-free water (Qiagen) was used for PCR reactions and cloning. PCR reactions were carried out using Phusion U Hot Start DNA polymerase (Thermo Fisher Scientific). Plasmids and SPs were cloned by USER assembly according to the manufacturer's instructions ${ }^{28}$. For antibodies and antigens used in this work, synthesized gBlock gene fragments were obtained from Integrated DNA Technologies. E. coli native genes were amplified directly from genomic DNA. Plasmids were cloned and amplified using Turbo (New England BioLabs) cells. Plasmid DNA was amplified for sequencing purposes using the Illustra Templiphi 100 Amplification Kit (GE Healthcare Life Sciences); SPs were amplified by PCR using primers AB1793 and AB1396. Phages were sequenced using primers AR007, MM1081, MM1082, TW629, and TW1243. All primer sequences can be found in Supplementary Table 4 . Sanger sequencing was used to confirm all plasmid sequences and to characterize SPs. Phage cloning and phage titer determination was carried out in strain S2208 ${ }^{28}$. Clone-specific sanger sequencing data for phages sequenced can be found in the Source data spreadsheet.

Plasmids and phage used in this work can be found in Supplementary Tables 1-3. Antibiotic (Gold Biotechnology) working concentrations were as follows: carbenicillin $50 \mu \mathrm{g} / \mathrm{mL}$, spectinomycin $50 \mu \mathrm{g} / \mathrm{mL}$, chloramphenicol $25 \mu \mathrm{g} / \mathrm{mL}$, kanamycin $50 \mu \mathrm{g} / \mathrm{mL}$, tetracycline $10 \mu \mathrm{g} / \mathrm{mL}$, streptomycin $50 \mu \mathrm{g} / \mathrm{mL}$.

\section{Preparation and transformation of competent cells. To prepare chemically} competent cells of strains S536, S1367 and S2208, overnight cultures were grown from single colonies and diluted 500 -fold into $10 \mathrm{~mL}$ of $2 \mathrm{xYT}$ media (United States Biologicals) supplemented with appropriate antibiotics. Cells were grown at $37^{\circ} \mathrm{C}$ with $230 \mathrm{RPM}$ shaking to $\mathrm{OD}_{600}=0.4-0.6$ and pelleted by centrifugation at $4000 \mathrm{~g}$ for $10 \mathrm{~min}$ at $4{ }^{\circ} \mathrm{C}$. The cell pellet was then resuspended in $500 \mu \mathrm{L}$ TSS (LB media supplemented with $2.5 \% \mathrm{v} / \mathrm{v}$ DMSO, 5\% w/v PEG 3350, and $10 \mathrm{mM} \mathrm{MgCl}_{2}$ ). For transformations, $50 \mu \mathrm{L}$ of competent cells were added to $1 \mu \mathrm{L}$ plasmid in $50 \mu \mathrm{L}$ prechilled $\mathrm{KCM}\left(100 \mathrm{mM} \mathrm{KCl}, 30 \mathrm{mM} \mathrm{CaCl}_{2}\right.$, and $50 \mathrm{mM} \mathrm{MgCl}_{2}$ in $\left.\mathrm{H}_{2} \mathrm{O}\right)$, incubated on ice for $15 \mathrm{~min}$, heat shocked at $42{ }^{\circ} \mathrm{C}$ for $90 \mathrm{~s}$ and incubated on ice $2 \mathrm{~min}$ prior to recovery.

To prepare electrocompetent cells of strains S1021, S536, S1367, single colonies or glycerol stocks were grown up overnight and diluted 500 -fold in 2xYT plus appropriate antibiotics. $10 \mathrm{~mL}$ of cells at $\mathrm{OD}_{600} 0.3-0.4$ were pelleted by centrifugation at $4000 \mathrm{~g}$ for $10 \mathrm{~min}$ at $4{ }^{\circ} \mathrm{C}$. The cell pellet was resuspended in $1 \mathrm{~mL}$ ice-cold $10 \%$ glycerol and washed $3 \mathrm{X}$ with $1 \mathrm{~mL}$ ice-cold glycerol, pelleting at $10,000 \mathrm{~g}$ for $1 \mathrm{~min}$ at $4^{\circ} \mathrm{C}$ between washes and maintaining cells on ice between spins. The pellet was resuspended in $500 \mu \mathrm{L}$ ice-cold $10 \%$ glycerol and the resulting mixture used fresh or else stored at $-80^{\circ} \mathrm{C}$. For transformation, $1 \mu \mathrm{L}$ each of up to three plasmids was added directly to $50 \mu \mathrm{L}$ of electrocompetent cells prior to electroporation in pre-chilled cuvettes (Bio-Rad).

Cells were recovered for $1 \mathrm{~h}$ at $37^{\circ} \mathrm{C}$ with shaking at $230 \mathrm{RPM}$ in $1 \mathrm{~mL}$ of SOC media (New England BioLabs) and streaked on 2xYT media $+1.5 \%$ agar (United States Biologicals) plates containing the appropriate antibiotics before incubation at $37^{\circ} \mathrm{C}$ for $12-18 \mathrm{~h}$

E. coli strains. All luminescence assays and evolution experiments were carried out in E. coli strains S536 and S1367. These strains were engineered from PACE strains
$\mathrm{S} 1030^{30}$ and $\mathrm{S} 2060^{35}$, respectively, using Lambda Red recombineering ${ }^{82}$ to replace the E. coli native CadCBA operon with a kanamycin resistance cassette. Chemically competent host cells of strain S1021 ${ }^{29}$ were transformed with plasmid pKD119 as described above. Primers MM557 and MM559 with 5' homology to regions of the genome flanking the cadCBA operon, were used to amplify the kanamycin resistance cassette from plasmid pKD13. The PCR product was gel-purified and transformed into $500 \mu \mathrm{L} \mathrm{S1021}+$ pKD119 cells by electroporation and recovered overnight at $37^{\circ} \mathrm{C}$ with shaking at $230 \mathrm{RPM}$ in $4 \mathrm{~mL}$ SOC, then plated on $2 \mathrm{xYT}+1.5 \%$ agar + kanamycin and incubated at $37^{\circ} \mathrm{C}$ for $16 \mathrm{~h}$.

Insertion of the kanamycin resistance cassette was verified by colony PCR using primers MM558 and MM560. Successful colonies were inoculated into 2xYT + kanamycin and grown up at $37^{\circ} \mathrm{C}$ for $5 \mathrm{~h}$ before plating in parallel on $2 \mathrm{xYT}+1.5 \%$ agar + kanamycin or tetracycline to verify successful curing of pKD119. Successful cultures were incubated in $2 \mathrm{xYT}+$ kanamycin for $2 \mathrm{~h}$ at $37^{\circ} \mathrm{C}$ with the addition of $1 \mu \mathrm{L}$ of F-plasmid donor culture, $\mathrm{S} 1030^{30}$ or $\mathrm{S} 2060^{35}$, and streaked on $2 \mathrm{xYT}+1.5 \%$ agar + kanamycin, tetracycline and streptomycin. Since the loss of the cadCBA operon is associated with a slight fitness cost, $\triangle$ cadCBA cells were maintained with kanamycin throughout subsequent work to safeguard against contamination by strains lacking the $\triangle$ cadCBA deletion.

Luciferase transcriptional activation assay. S536 and S1367 cells were transformed with APs and CPs as indicated in Supplementary table 2. Freshly saturated cultures of single colonies grown in Davis Rich Media ${ }^{19}$ (DRM) plus maintenance antibiotics were diluted 500-fold into DRM media with maintenance antibiotics in a 96-well deep-well plate (Axygen) and induced with indicated concentrations of arabinose (Gold Biotechnology) before incubation for $2 \mathrm{~h}$ at $37^{\circ} \mathrm{C}$ with shaking at 230 RPM. $150 \mu \mathrm{L}$ of cells per well were then transferred to a 96-well black-walled clear-bottomed plate with a transparent lid (Costar). $600 \mathrm{~nm}$ absorbance and luminescence were read at 15-min intervals over an 8-h kinetic cycle with shaking at 230 RPM between reads using a Tecan Spark multimode microplate reader (Tecan). Single read data were taken at peak luminescence value $(4-5 \mathrm{~h}$ postinduction). $\mathrm{OD}_{600}$-normalized luminescence values were determined by dividing raw luminescence by background-subtracted (DRM only) $600 \mathrm{~nm}$ absorbance.

For phage-induced luciferase timecourse assay, S536 and S2060 cells were transformed with APs and diluted in DRM as described above. Cells were grown to an $\mathrm{OD}_{600}$ of 0.4 and were inoculated with selection phage at an initial titer of $5 \times 10^{4} \mathrm{pfu} / \mathrm{mL}$. $150 \mu \mathrm{L}$ of cells per well were immediately transferred to a plate for luminescence and optical density reading in a kinetic cycle as described above.

Phage propagation assay. S536 and S1367 cells were transformed with the AP(s) of interest as described above. Overnight cultures of single colonies grown in 2xYT media supplemented with maintenance antibiotics were diluted 1000 -fold into DRM media with maintenance antibiotics and grown at $37^{\circ} \mathrm{C}$ with shaking at 230 $\mathrm{RPM}$ to $\mathrm{OD}_{600} 0.4$ exactly. Cells were infected with SP at an initial titer of $5 \times 10^{4} \mathrm{pfu} \mathrm{mL}^{-1}$. Cells were incubated $16-18 \mathrm{~h}$ at $37^{\circ} \mathrm{C}$ with shaking at $230 \mathrm{RPM}$ then centrifuged at $10,000 \mathrm{~g}$ for $2 \mathrm{~min}$ and the supernatant stored at $4{ }^{\circ} \mathrm{C}$.

Plaque assay. Saturated cultures of single colonies of strain S2208 grown in 2xYT media plus maintenance antibiotics were diluted 1000 -fold into fresh 2xYT media with maintenance antibiotics and grown at $37^{\circ} \mathrm{C}$ with shaking at $230 \mathrm{RPM}$ to $\mathrm{OD}_{600} \sim 0.8$ before use. SPs were serially diluted 100 -fold (4 dilutions total) in $\mathrm{H}_{2} \mathrm{O}$. $10 \mu \mathrm{L}$ of phage dilution was added to $150 \mu \mathrm{L}$ of cells and immediately mixed with $1 \mathrm{~mL}$ of liquid $\left(55^{\circ} \mathrm{C}\right)$ top agar $(2 \mathrm{xYT}$ media $+0.6 \%$ agar) supplemented with $2 \%$ Bluo-gal (Gold Biotechnology). The mixture was then immediately pipetted onto one quadrant of a quartered Petri dish containing $2 \mathrm{~mL}$ of solidified bottom agar $(2 \mathrm{xYT}$ media $+1.5 \%$ agar, no antibiotics) and allowed to solidify. Plates were incubated at $37^{\circ} \mathrm{C}$ for $16-18 \mathrm{~h}$. Titers were reported as one significant figure prior to calculating ratios.

Phage-assisted continuous and non-continuous evolution. Cell preparation, PANCE, and PACE were carried out in $\mathrm{DRM}^{61}$. Chemically competent S536 or S1367 cells were transformed with AP(s) and DP6, plated on 2xYT media $+1.5 \%$ agar supplemented with $10 \mathrm{mM}$ glucose (to suppress induction of mutagenesis from the $\mathrm{P}_{\mathrm{BAD}}$ promoter) and maintenance antibiotics, and grown at $37^{\circ} \mathrm{C}$ for $16 \mathrm{~h}$. Colonies were picked into $500 \mu \mathrm{L}$ DRM in a 96-well deep-well plate, and serially diluted 10-fold twelve times in DRM. Typically, eight colonies were selected. The plate was sealed with porous film and colonies allowed to grow at $37^{\circ} \mathrm{C}$ with shaking at $230 \mathrm{RPM}$ for $16-18 \mathrm{~h}$.

For PACE, dilutions with $\mathrm{OD}_{600} \sim 0.4-0.8$ were then used to inoculate an $80 \mathrm{~mL}$ DRM chemostat. The chemostat was continuously diluted with fresh DRM at a rate of $\sim 1.5$ chemostat volumes/h, maintaining a volume of $60-80 \mathrm{~mL}$ and an $\mathrm{OD}_{600}$ value between 0.8 and $1.0^{17}$

Lagoons were continuously diluted from the chemostat culture at 1 lagoon volume/h and were induced with $10 \mathrm{mM}$ arabinose $\pm 50 \mathrm{ng} / \mathrm{mL}$ aTc as indicated, for at least $2 \mathrm{~h}$ prior to infection with SP. For novel PACE campaigns, SPs were plaqued as described above and purified from single plaques by growing up $\sim 8 \mathrm{~h}$ in fresh $2 \mathrm{xYT}$ media with maintenance antibiotics at $37^{\circ} \mathrm{C}$ with shaking at $230 \mathrm{RPM}$. For continuations of previous PACE runs at increased stringency, $20 \mu \mathrm{L}$ of lagoon samples from previous PACE endpoints were added to $2 \mathrm{~mL}$ of S2208 cells in 
mid-log growth phase and grown for $\sim 4 \mathrm{~h}$ in $2 \mathrm{xYT}$ media plus maintenance antibiotics at $37^{\circ} \mathrm{C}$ with shaking at $230 \mathrm{RPM}$. All selection phage cultures were centrifuged at $10,000 \mathrm{~g}$ for $2 \mathrm{~min}$ and passed through a $0.22-\mu \mathrm{m}$ PVDF Ultrafree centrifugal filter (Millipore) prior to use in PACE.

Lagoons were infected with purified SP at a starting titer of $10-10^{6} \mathrm{pfu} / \mathrm{mL}$ and maintained at a volume of $15 \mathrm{~mL}$ through the constant inflow of chemostat material and outflow of media waste at a rate of 0.5-3 lagoon volumes per hour. Arabinose and aTc concentrations within lagoons were maintained through constant inflow. 500- $\mu \mathrm{L}$ samples were taken at indicated times from lagoon waste lines. Samples were centrifuged at $10,000 \mathrm{~g}$ for $2 \mathrm{~min}$, and the supernatant was passed through a $0.22-\mu \mathrm{m}$ PVDF Ultrafree centrifugal filter (Millipore) and stored at $4^{\circ} \mathrm{C}$.

Selection phage titers were determined by plaque assays using S2208 cells. Four or eight single plaques were PCR amplified as described above to characterize lagoon phage.

For PANCE, host strain dilutions with $\mathrm{OD}_{600} \sim 0.4-0.8$ were further diluted to $50 \mathrm{~mL}$ in DRM plus appropriate antibiotics and grown up to $\mathrm{OD}_{600} \sim 0.4 .1 \mathrm{~mL}$ of cells were added to each well of a deep-well plate, allocating one well per replicate. Wells were induced with $10 \mathrm{mM}$ arabinose if mutagenesis/drift plasmid was present and were inoculated with phage at $10^{7} \mathrm{pfu} \mathrm{mL}^{-1}$ unless otherwise indicated. Plates were grown up $16 \mathrm{~h}$ at $37^{\circ} \mathrm{C}$ with shaking at $230 \mathrm{RPM}$. Plaques were amplified for characterization as described above. For restriction-enzymemediated phage characterization, $400 \mathrm{ng}$ PCR-amplified phage DNA was cleaved with $0.4 \mu \mathrm{L}$ Hinfl (New England Biolabs) according to manufacturer's instructions.

\section{Small-scale protein expression. BL21 DE3 cells (New England BioLabs) were} transformed with expression plasmids (EPs) according to the manufacturer's protocol. Single colonies were grown up overnight in $2 \mathrm{xYT}$ media plus maintenance antibiotics were diluted 1000 -fold into fresh $2 \mathrm{xYT}$ media $(2 \mathrm{~mL})$ with maintenance antibiotics and grown at $37^{\circ} \mathrm{C}$ with shaking at $230 \mathrm{RPM}$ to $\mathrm{OD}_{600} 0.4$. Cells were induced with $0.1 \mathrm{mM}$ isopropyl- $\beta$-D-thiogalactoside (IPTG; Gold Biotechnology) or other indicated concentration and grown for a further $4 \mathrm{~h}$ at $37^{\circ} \mathrm{C}$ with shaking at $230 \mathrm{RPM} .2 \mathrm{OD}_{600}$ units of culture were isolated by centrifugation at $8000 \mathrm{~g}$ for $2 \mathrm{~min}$. The resulting pellet was resuspended in $150 \mu \mathrm{L}$ B-per reagent (Thermo Fisher Scientific) supplemented with protease inhibitor cocktail (Roche) and incubated at $25^{\circ} \mathrm{C}$ for $15 \mathrm{~min}$ before centrifugation at $16,000 \mathrm{~g}$ for $2 \mathrm{~min}$. The supernatant was collected as the soluble fraction. The pellet was resuspended in an additional $150 \mu \mathrm{L}$ B-per reagent to obtain the insoluble fraction. To $37.5 \mu \mathrm{L}$ of each fraction was added $12.5 \mu \mathrm{L} 4 \mathrm{x}$ NuPage LDS sample buffer (Thermo Fisher Scientific). Fractions were vortexed and incubated at $95^{\circ} \mathrm{C}$ for $10 \mathrm{~min} .12 \mu \mathrm{L}$ (soluble fraction) or $5 \mu \mathrm{L}$ (insoluble fraction) was loaded per well of a Bolt $4-12 \%$ Bis-Tris Plus (Thermo Fisher Scientific) pre-cast gel. $5 \mu \mathrm{L}$ of Precision Plus Protein Dual Color Standard (Bio-Rad) was used as a reference. Samples were separated by electrophoresis at $200 \mathrm{~V}$ for $30 \mathrm{~min}$ in Bolt MES SDS running buffer (Thermo Fisher Scientific). Gels were stained with InstantBlue reagent (Expedeon) for $-16 \mathrm{~h}$ and destained for $1 \mathrm{~h}$ in water before imaging with a G:Box Chemi XRQ (Syngene).

Periplasmic extraction. Periplasmic extraction was carried out as previously described ${ }^{83}$. Briefly, $100 \mathrm{~mL}$ of cells at $\mathrm{OD}_{600}=\sim 1$ were pelleted by centrifugation at $3000 \mathrm{~g}$, drained, and carefully resuspended in $1 \mathrm{~mL}$ TSE buffer $(200 \mathrm{mM}$ Tris$\mathrm{HCl} \mathrm{pH} \mathrm{8.0,500} \mathrm{mM} \mathrm{sucrose,} 1 \mathrm{mM}$ EDTA) plus protease inhibitor cocktail (Roche). Cell pellets were incubated on ice for $30 \mathrm{~min}$ and supernatant (periplasm extract) was separated from cell pellet (spheroplast) by centrifugation at $16,000 \mathrm{~g}$ for $30 \mathrm{~min}$ at $4 \mathrm{C}$. Samples were analyzed by SDS-PAGE and Western blot.

Western blot analysis. Following SDS-PAGE, proteins were transferred to a PVDF membrane using an iBlot 2 Gel Transfer Device (Thermo Fisher Scientific) according to the manufacturer's protocol. The membrane was blocked in SuperBlock Blocking Buffer (Thermo Fisher Scientific) for $1 \mathrm{~h}$ at room temperature, then incubated overnight at $4{ }^{\circ} \mathrm{C}$ in SuperBlock Blocking Buffer (Thermo Fisher Scientific) plus one or more of the following, as indicated: mouse anti-6xHis (abcam ab18184; 1:2000 dilution), mouse anti-c-ABL (Sigma-Aldrich A5844; 1:2000 dilution), mouse anti-MBP (abcam ab65, 1:5000 dilution) and rabbit antiGroEL (Sigma-Aldrich G6532; 1:20,000 dilution). If both primary and loading control antibodies were mouse-derived, as in Supplementary Fig. 6g, membrane was cut according to expected MW of target and membrane halves were incubated separately in primary antibodies, as indicated. The membrane was washed $3 \mathrm{x}$ with TBST (TBS $+0.5 \%$ Tween-20) for $10 \mathrm{~min}$ each at room temperature, then incubated with IRDye-labeled secondary antibodies goat anti-mouse 680RD (LI-COR 926-68070) and donkey anti-rabbit 800CW (LI-COR 926-32213) diluted 1:5000 for $1 \mathrm{~h}$ at $25^{\circ} \mathrm{C}$. The membrane was washed $3 \mathrm{x}$ with TBS as before. Imaging was performed using the Odyssey Imaging System (LI-COR).

Band densities were quantified using ImageJ and normalized to reference bands to control for loading. Uncropped blot images can be found in Supplementary Figs. 9, 15 and 16.

Large-scale protein expression and purification. BL21 DE3 cells transformed with EPs of interest were grown in LB or 2xYT media containing maintenance antibiotics overnight from single colonies. Cultures were diluted 1000 -fold into fresh $2 x Y T$ media $(1 \mathrm{~L})$ with appropriate antibiotics and grown up at $37^{\circ} \mathrm{C}$ with shaking at $230 \mathrm{RPM}$ to $\mathrm{OD}_{600} \sim 0.4-0.5$. Cells were induced with $50 \mathrm{uM} \mathrm{IPTG}$ and grown for a further $16-18 \mathrm{~h}$ at $16^{\circ} \mathrm{C}$ with shaking at $200 \mathrm{RPM}$. Cells were isolated by centrifugation at $8000 \mathrm{~g}$ for $10 \mathrm{~min}$ and washed $1 \mathrm{x}$ with $20 \mathrm{~mL}$ TBS $(20 \mathrm{mM}$ Tris$\mathrm{Cl}, 500 \mathrm{mM} \mathrm{NaCl}, \mathrm{pH}$ 7.5). The resulting pellet was resuspended in $12 \mathrm{~mL}$ B-per reagent supplemented with EDTA-free protease inhibitor cocktail (Roche) and incubated on ice for $30 \mathrm{~min}$ with regular vortexing, before centrifugation at $16,000 \mathrm{~g}$ for $18 \mathrm{~min}$. The supernatant was decanted into a $50 \mathrm{~mL}$ conical tube and incubated with $1 \mathrm{~mL}$ of TALON Cobalt (Clontech) resin at $4{ }^{\circ} \mathrm{C}$ with constant agitation for $2 \mathrm{~h}$, after which the resin was isolated by centrifugation at $500 \mathrm{~g}$ for $5 \mathrm{~min}$. The supernatant was decanted, and the resin resuspended in $4 \mathrm{~mL}$ binding buffer $\left(50 \mathrm{mM} \mathrm{NaH}_{2} \mathrm{PO}_{4}, 300 \mathrm{mM} \mathrm{NaCl}, 20 \mathrm{mM}\right.$ imidazole, $\mathrm{pH}$ 7.8) and transferred to a column. The resin was washed $4 \mathrm{x}$ with $4 \mathrm{~mL}$ binding buffer before protein was eluted with $2 \times 1 \mathrm{~mL}$ of binding buffer containing increasing concentrations of imidazole (50-300 $\mathrm{mM}$ in $50 \mathrm{mM}$ increments). The fractions were analyzed by SDS-PAGE. Combined pure fractions were buffer-exchanged with TBS and concentrated using an Amicon Ultra-15 centrifugal filter unit (10,000 molecular weight cutoff; Millipore), then stored at $4^{\circ} \mathrm{C}$ for up to one week or else snapfrozen in liquid nitrogen for $-80^{\circ} \mathrm{C}$ storage. Total protein was quantified using a BCA protein assay kit (Pierce) using BSA standards (Bio-Rad). Quantification of specific bands, where necessary, was carried out by gel densitometry using ImageJ software with comparison to reference lanes loaded with known quantities of BSA (Bio-Rad).

ELISA. Pre-blocked high-capacity streptavidin-coated 96-well clear plates (Pierce) were washed $3 \mathrm{X}$ with $200 \mathrm{ul} /$ well TBST and incubated overnight at $4 \mathrm{C}$ with purified biotin-tagged protein (Her2, TGFB1, AcroBiosystems; H98 peptide, biotin-GGGGSLLGPYELWELSH, GenScript Custom Peptide) diluted as indicated in TBS. After overnight incubation, wells were washed 3X with 200ul/well TBST and incubated at room temperature for $2 \mathrm{~h}$ with $25 \mathrm{ug} / \mathrm{mL}$ purified antibody fragments in TBS, $50 \mu \mathrm{L}$ per well. Wells were washed $3 \mathrm{X}$ with $200 \mathrm{ul} /$ well TBST and incubated for a further $45 \mathrm{~min}$ with protein a-HRP (Thermo Fisher Scientific $101023,1: 2000$ dilution) in TBS. Finally, wells were washed 4X with 200ul/well TBST, then developed with $50 \mu \mathrm{L} /$ well 1-Step Ultra TMB-ELISA Substrate (Thermo Fisher Scientific) for 90 seconds. Quenching was carried out with $50 \mu \mathrm{L} /$ well $2 \mathrm{M} \mathrm{H}_{2} \mathrm{SO}_{4}$ and $450 \mathrm{~nm}$ absorbance was read using a Tecan Spark multimode microplate reader. Values were normalized by subtracting the mean value at $0 \mathrm{nM}$ antigen for each variant and dividing all values by the maximum mean value of the unmutated TR control. $\mathrm{EC}_{50}$ values were calculated using a sigmoidal 4-point linear regression in Prism 8.

Microscale thermophoresis. MST was carried out using the Monolith NT.115 system (Nanotemper) according to the manufacturer's instructions. H98 peptide (GenScript) was resuspended in DMSO and diluted in TBS-T to a final concentration of $6.25 \%$ DMSO. Trastuzumab and variant scFvs were diluted in TBS-T to a final concentration of $5 \mathrm{nM}$ and fluorophore-tagged with cy3conjugated anti-6XH antibody (Rockland Antibodies \& Assays) at a 1:1 molar ratio. Reads were carried out using Monolith.NT automated capillary chips (Nanotemper). Data were analyzed with built-in MO.Control and MO.Affinity Analysis software, version 2.3.

Growth timecourse assay. For phage-based growth timecourse assays, S1367 cells were transformed with permissive accessory plasmid $\mathrm{pJC} 175 \mathrm{e}^{19}$. Freshly saturated cultures of single colonies grown in $\mathrm{DRM}^{19}$ media plus maintenance antibiotics were diluted 1000-fold into DRM media with maintenance antibiotics until $\mathrm{OD}_{600}$ $\sim 0.1$ was reached. Biological replicates were infected with phage at indicated initial titers, and $150 \mu \mathrm{L}$ of cells per well were immediately transferred to a 96-well blackwalled clear-bottomed plate with a transparent lid (Costar). $600 \mathrm{~nm}$ absorbance and luminescence were read at 10-min intervals over a 9-h kinetic cycle with shaking at 230 RPM between reads using a Tecan Spark multimode microplate reader (Tecan)

For plasmid-based growth timecourse assays, S1367 cells were transformed with pJC175e and CPs as indicated in Supplementary table 2. Freshly saturated cultures of single colonies grown in DRM ${ }^{19}$ media plus maintenance antibiotics were diluted 500-fold into DRM media with maintenance antibiotics in a 96-well deepwell plate (Axygen) and induced with indicated concentrations of arabinose (Gold Biotechnology) before incubation for $2 \mathrm{~h}$ at $37^{\circ} \mathrm{C}$ with shaking at $230 \mathrm{RPM} .150 \mu \mathrm{L}$ of cells per well were then transferred to a 96-well black-walled clear-bottomed plate with a transparent lid (Costar). $600 \mathrm{~nm}$ absorbance and luminescence were read at 10-min intervals over a 9-h kinetic cycle with shaking at 230 RPM between reads using a Tecan Spark multimode microplate reader (Tecan).

Protein melt temperature assay. Melt temperatures were determined using the Protein Thermal Shift Dye Kit (Life Technologies) according to the manufacturer's protocols. A CFX96 Real-Time PCR Detection System (Bio-Rad) was used to monitor fluorescence. 
Reporting summary. Further information on research design is available in the Nature Research Reporting Summary linked to this article.

\section{Data availability}

Materials such as plasmids and maps will be made available upon reasonable request. Cited crystal structure data are available in the Protein Database under entry IDS 1J85 (https://www.rcsb.org/structure/1J85) and 1N8Z (https://www.rcsb.org/structure/1N8Z). Source data are provided with this paper. No code was used in this manuscript. No large datasets were generated in this manuscript. Source data are provided with this paper.

Received: 7 August 2021; Accepted: 27 September 2021; Published online: 13 October 2021

\section{References}

1. Awwad, S. \& Angkawinitwong, U. Overview of antibody drug delivery. Pharmaceutics 10, 83 (2018)

2. Lu, R.-M. et al. Development of therapeutic antibodies for the treatment of diseases. J. Biomed. Sci. 27, 1-30 (2020).

3. Ahmad, Z. A. et al. scFv Antibody: Principles and Clinical Application. Clin. Developmental Immunol. 2012, 980250 (2012).

4. Smith, G. P. Filamentous fusion phage: novel expression vectors that display cloned antigens on the virion surface. Science 228, 1315-1317 (1985).

5. Winter, G. \& Milstein, C. Man-made antibodies. Nature 349, 293-299 (1991).

6. Pollock, S. B. et al. Highly multiplexed and quantitative cell-surface protein profiling using genetically barcoded antibodies. Proc. Natl Acad. Sci. 115, 2836 (2018).

7. Liu, C. C. et al. Protein evolution with an expanded genetic code. Proc. Natl Acad. Sci. USA 105, 17688-17693 (2008).

8. Jeong, K. J., Seo, M. J., Iverson, B. L. \& Georgiou, G. APEx 2-hybrid, a quantitative protein-protein interaction assay for antibody discovery and engineering. Proc. Natl Acad. Sci. 104, 8247 (2007)

9. Waraho-Zhmayev, D., Meksiriporn, B., Portnoff, A. D. \& DeLisa, M. P. Optimizing recombinant antibodies for intracellular function using hitchhiker-mediated survival selection. Protein Eng., Des. Selection 27, 351-358 (2014).

10. Robinson, M.-P. et al. Isolation of full-length IgG antibodies from combinatorial libraries expressed in the cytoplasm of Escherichia coli. bioRxiv https://doi.org/10.1101/2020.05.09.085944 (2020).

11. Lombana, T. N., Dillon, M., Bevers, J. 3rd \& Spiess, C. Optimizing antibody expression by using the naturally occurring framework diversity in a live bacterial antibody display system. Sci. Rep. 5, 17488 (2015).

12. Moghaddam-Taaheri, P., Ikonomova, S. P., Gong, Z., Wisniewski, J. Q. \& Karlsson, A. J. Bacterial Inner-membrane Display for Screening a Library of Antibody Fragments. JoVE e54583, https://doi.org/10.3791/54583 (2016).

13. Wellner, A. et al. Rapid generation of potent antibodies by autonomous hypermutation in yeast. bioRxiv https://doi.org/10.1101/2020.11.11.378778 (2020).

14. Morrison, M. S., Podracky, C. J. \& Liu, D. R. The developing toolkit of continuous directed evolution. Nat. Chem. Biol. 16, 610-619 (2020).

15. Badran, A. H. \& Liu, D. R. In vivo continuous directed evolution. Curr. Opin. Chem. Biol. 24, 1-10 (2015).

16. Packer, M. S. \& Liu, D. R. Methods for the directed evolution of proteins. Nat. Rev. Genet. 16, 379-394 (2015).

17. Arnold, F. H. Directed evolution: bringing new chemistry to life. Angew. Chem. Int Ed. Engl. 57, 4143-4148 (2018).

18. Alfaleh, M. A. et al. Phage display derived monoclonal antibodies: from bench to bedside. Front. Immunol. 11, 1986 (2020).

19. Esvelt, K. M., Carlson, J. C. \& Liu, D. R. A system for the continuous directed evolution of biomolecules. Nature 472, 499-503 (2011).

20. Hornsby, M. et al. A high through-put platform for recombinant antibodies to folded proteins. Mol. Cell Proteom. 14, 2833-2847 (2015).

21. Tassone, E. E. et al. Sequencing, de novo assembly and annotation of a pink bollworm larval midgut transcriptome. Gigascience 5, 28 (2016).

22. $\mathrm{Pu}$, J., Zinkus-Boltz, J. \& Dickinson, B. C. Evolution of a split RNA polymerase as a versatile biosensor platform. Nat. Chem. Biol. 13, 432 (2017).

23. Wong, B. G., Mancuso, C. P., Kiriakov, S., Bashor, C. J. \& Khalil, A. S. Precise, automated control of conditions for high-throughput growth of yeast and bacteria with eVOLVER. Nat. Biotechnol. 36, 614-623 (2018).

24. Berman, C. M. et al. An adaptable platform for directed evolution in human cells. J. Am. Chem. Soc. 140, 18093-18103 (2018).

25. English, J. G. et al. VEGAS as a platform for facile directed evolution in mammalian cells. Cell 178, 748-761.e717 (2019).
26. Ravikumar, A., Arzumanyan, G. A., Obadi, M. K. A., Javanpour, A. A. \& Liu, C. C. Scalable, continuous evolution of genes at mutation rates above genomic error thresholds. Cell 175, 1946-1957.e1913 (2018).

27. Crook, N. et al. In vivo continuous evolution of genes and pathways in yeast. Nat. Commun. 7, https://doi.org/10.1038/ncomms13051 (2016).

28. Badran, A. H. et al. Continuous evolution of Bacillus thuringiensis toxins overcomes insect resistance. Nature 533, 58-63 (2016).

29. Badran, A. H. \& Liu, D. R. Development of potent in vivo mutagenesis plasmids with broad mutational spectra. Nat. Commun. 6, 8425 (2015).

30. Carlson, J. C., Badran, A. H., Guggiana-Nilo, D. A. \& Liu, D. R. Negative selection and stringency modulation in phage-assisted continuous evolution. Nat. Chem. Biol. 10, 216-222 (2014).

31. Dickinson, B. C., Packer, M. S., Badran, A. H. \& Liu, D. R. A system for the continuous directed evolution of proteases rapidly reveals drug-resistance mutations. Nat. Commun. 5, 5352 (2014).

32. Packer, M. S., Rees, H. A. \& Liu, D. R. Phage-assisted continuous evolution of proteases with altered substrate specificity. Nat. Commun. 8, https://doi.org/ 10.1038/s41467-017-01055-9 (2017).

33. Blum, T. R. et al. Phage-assisted evolution of botulinum neurotoxin proteases with reprogrammed specificity. Science 371, 803-810 (2021).

34. Bryson, D. I. et al. Continuous directed evolution of aminoacyl-tRNA synthetases. Nat. Chem. Biol. 13, 1253 (2017)

35. Hubbard, B. P. et al. Continuous directed evolution of DNA-binding proteins to improve TALEN specificity. Nat. Methods 12, 939-942 (2015).

36. $\mathrm{Hu}, \mathrm{J}$. H. et al. Evolved Cas 9 variants with broad PAM compatibility and high DNA specificity. Nature 556, 57-63 (2018).

37. Miller, S. M. et al. Continuous evolution of SpCas9 variants compatible with non-G PAMs. Nat. Biotechnol. https://doi.org/10.1038/s41587-020-0412-8 (2020).

38. Roth, T. B., Woolston, B. M., Stephanopoulos, G. \& Liu, D. R. Phage-assisted evolution of bacillus methanolicus methanol dehydrogenase 2. ACS Synth Biol. https://doi.org/10.1021/acssynbio.8b00481 (2019).

39. Wang, T., Badran, A. H., Huang, T. P. \& Liu, D. R. Continuous directed evolution of proteins with improved soluble expression. Nat. Chem. Biol. https://doi.org/10.1038/s41589-018-0121-5 (2018).

40. Thuronyi, B. W. et al. Continuous evolution of base editors with expanded target compatibility and improved activity. Nat. Biotechnol. 37, 1070-1079 (2019).

41. Richter, M. F. et al. Phage-assisted evolution of an adenine base editor with improved Cas domain compatibility and activity. Nat. Biotechnol. https:// doi.org/10.1038/s41587-020-0453-z (2020)

42. Johnston, C. W., Badran, A. H. \& Collins, J. J. Continuous bioactivitydependent evolution of an antibiotic biosynthetic pathway. Nat. Commun. 11 4202 (2020).

43. Proba, K., Wörn, A., Honegger, A. \& Plückthun, A. Antibody scFv fragments without disulfide bonds, made by molecular evolution. J. Mol. Biol. 275 , 245-253 (1998).

44. Liu, T. et al. Enhancing protein stability with extended disulfide bonds. Proc Natl Acad. Sci. 113, 5910 (2016).

45. Tanaka, T. \& Rabbitts, T. H. Intrabodies based on intracellular capture frameworks that bind the RAS protein with high affinity and impair oncogenic transformation. EMBO J. 22, 1025-1035 (2003).

46. Colby, D. W. et al. Potent inhibition of huntingtin aggregation and cytotoxicity by a disulfide bond-free single-domain intracellular antibody. Proc. Natl Acad. Sci. USA 101, 17616-17621 (2004).

47. Colby, D. W. et al. Development of a Human Light Chain Variable Domain (VL) Intracellular Antibody Specific for the Amino Terminus of Huntingtin via Yeast Surface Display. J. Mol. Biol. 342, 901-912 (2004).

48. Gennari, F. et al. Direct phage to intrabody screening (DPIS): demonstration by isolation of cytosolic intrabodies against the TES1 site of Epstein Barr virus latent membrane protein 1 (LMP1) that block NF-kappaB transactivation. J. Mol. Biol. 335, 193-207 (2004).

49. Asial, I. et al. Engineering protein thermostability using a generic activity-independent biophysical screen inside the cell. Nat. Commun. 4, 2901 (2013).

50. Matos, C. F. et al. Efficient export of prefolded, disulfide-bonded recombinant proteins to the periplasm by the Tat pathway in Escherichia coli CyDisCo strains. Biotechnol. Prog. 30, 281-290 (2014).

51. Müller, A. et al. Nonnative disulfide bond formation activates the $\sigma 32$ dependent heat shock response in Escherichia coli. J. Bacteriol. 195, 2807 (2013).

52. Ramm, K., Gehrig, P. \& Plückthun, A. Removal of the conserved disulfide bridges from the scfv fragment of an antibody: effects on folding kinetics and aggregation. J. Mol. Biol. 290, 535-546 (1999)

53. Chang, H.-J. et al. A modular receptor platform to expand the sensing repertoire of bacteria. ACS Synth. Biol. 7, 166-175 (2018).

54. Kuper, C. \& Jung, K. CadC-mediated activation of the cadBA promoter in Escherichia coli. J. Mol. Microbiol. Biotechnol. 10, 26-39 (2005). 
55. Russ, W. P. \& Engelman, D. M. TOXCAT: A measure of transmembrane helix association in a biological membrane. Proc. Natl Acad. Sci. 96, 863 (1999).

56. Schlundt, A. et al. Structure-function analysis of the DNA-binding domain of a transmembrane transcriptional activator. Sci. Rep. 7, 1051 (2017).

57. Wojcik, J. et al. A potent and highly specific FN3 monobody inhibitor of the Abl SH2 domain. Nat. Struct. Mol. Biol. 17, 519-527 (2010).

58. Inouye, H., Barnes, W. \& Beckwith, J. Signal sequence of alkaline phosphatase of Escherichia coli. J. Bacteriol. 149, 434-439 (1982).

59. Mallam, A. L., Morris, E. R. \& Jackson, S. E. Exploring knotting mechanisms in protein folding. Proc. Natl Acad. Sci. USA 105, 18740-18745 (2008).

60. Mallam, A. L. \& Jackson, S. E. The dimerization of an alpha/beta-knotted protein is essential for structure and function. Structure 15, 111-122 (2007).

61. Miller, S. M., Wang, T. \& Liu, D. R. Phage-assisted continuous and noncontinuous evolution. Nat. Protocols 15, 4101-4127 (2020).

62. Lim, K. et al. Structure of the YibK methyltransferase from Haemophilus influenzae (HI0766): a cofactor bound at a site formed by a knot. Proteins 51, 56-67 (2003)

63. Frenzel, A., Hust, M. \& Schirrmann, T. Expression of recombinant antibodies. Front. Immunol. 4, https://doi.org/10.3389/fimmu.2013.00217 (2013).

64. der Maur, A. A. et al. Direct in vivo screening of intrabody libraries constructed on a highly stable single-chain framework. J. Biol. Chem. 277, 45075-45085 (2002).

65. Leder, L. et al. Spectroscopic, calorimetric, and kinetic demonstration of conformational adaptation in peptide-antibody recognition. Biochemistry 34, 16509-16518 (1995).

66. Stevens, A. J. et al. Design of a split intein with exceptional protein splicing activity. J. Am. Chem. Soc. 138, 2162-2165 (2016).

67. Zettler, J., Schütz, V. \& Mootz, H. D. The naturally split Npu DnaE intein exhibits an extraordinarily high rate in the protein trans-splicing reaction. FEBS Lett. 583, 909-914 (2009).

68. Albrecht, H. et al. Production of soluble ScFvs with C-terminal-free thiol for site-specific conjugation or stable dimeric ScFvs on demand. Bioconjugate Chem. 15, 16-26 (2004)

69. Arndt, K. M., Müller, K. M. \& Plückthun, A. Factors influencing the dimer to monomer transition of an antibody single-chain Fv fragment. Biochemistry 37, 12918-12926 (1998).

70. Molina, M. A. et al. Trastuzumab (Herceptin), a humanized Anti-HER2 receptor monoclonal antibody, inhibits basal and activated HER2 ectodomain cleavage in breast cancer cells. Cancer Res. 61, 4744-4749 (2001)

71. Friedman, M. et al. Engineering and characterization of a bispecific HER2 $\times$ EGFRbinding affibody molecule. Biotechnol. Appl. Biochem. 54, 121-131 (2009).

72. Bostrom, J. et al. Variants of the antibody herceptin that interact with HER2 and VEGF at the antigen binding site. Science 323, 1610-1614 (2009).

73. Nielsen, U. et al. MM-111: a novel bispecific antibody targeting ErbB3 with potent anti-tumor activity in ErbB2 over-expressing malignancies. Cancer Res. 69, 4166 (2009).

74. Jiang, B. et al. A novel peptide isolated from a phage display peptide library with trastuzumab can mimic antigen epitope of HER-2. J. Biol. Chem. 280, 4656-4662 (2005).

75. Cho, H. S. et al. Structure of the extracellular region of HER2 alone and in complex with the Herceptin Fab. Nature 421, 756-760 (2003).

76. Wörn, A. \& Plückthun, A. An intrinsically stable antibody scFv fragment can tolerate the loss of both disulfide bonds and fold correctly. FEBS Lett. 427, 357-361 (1998).

77. Krauss, J., Arndt, M. A., Martin, A. C., Liu, H. \& Rybak, S. M. Specificity grafting of human antibody frameworks selected from a phage display library: generation of a highly stable humanized anti-CD22 single-chain Fv fragment. Protein Eng. 16, 753-759 (2003).

78. Seo, M. J. et al. Engineering antibody fragments to fold in the absence of disulfide bonds. Protein Sci.: Publ. Protein Soc. 18, 259-267 (2009).

79. Decad, G. M. \& Nikaido, H. Outer membrane of gram-negative bacteria. XII. Molecular-sieving function of cell wall. J. Bacteriol. 128, 325-336 (1976).
80. Wilks, J. C. \& Slonczewski, J. L. pH of the cytoplasm and periplasm of escherichia coli: rapid measurement by green fluorescent protein fluorimetry. J. Bacteriol. 189, 5601-5607 (2007).

81. Stefanick, J. F., Kiziltepe, T., Handlogten, M. W., Alves, N. J. \& Bilgicer, B. Enhancement of antibody selectivity via bicyclic complex formation. J. Phys. Chem. Lett. 3, 598-602 (2012).

82. Datsenko, K. A. \& Wanner, B. L. One-step inactivation of chromosomal genes in Escherichia coli K-12 using PCR products. Proc. Natl Acad. Sci. USA 97, 6640-6645 (2000)

83. Quan, S., Hiniker, A., Collet, J. F. \& Bardwell, J. C. Isolation of bacteria envelope proteins. Methods Mol. Biol. 966, 359-366 (2013).

\section{Acknowledgements}

This work was supported by NIH U01 AI142756, R01 EB031172, R01 EB027793, and R35 GM118062; and HHMI. We thank Ahmed Badran for helpful comments and Alvin Hsu for assistance with MST.

\section{Author contributions}

M.M. designed the research, performed the experiments, analyzed data, and wrote the manuscript. T.W. designed the research, performed the experiments and analyzed data A.R. designed the research, performed the experiments and analyzed data. C.H. performed the experiments. D.R.L. designed and supervised the research and wrote the manuscript.

\section{Competing interests}

M.S.M., T.W., A.R., C.H., and D.R.L. have filed a provisional patent application through the Broad Institute on the periplasmic PACE system and related improvements. D.R.L. is a co-founder and consultant of Chroma Medicine and Resonance Medicine, companies that may use PACE technologies.

\section{Additional information}

Supplementary information The online version contains supplementary material available at https://doi.org/10.1038/s41467-021-26279-8.

Correspondence and requests for materials should be addressed to David R. Liu.

Peer review information Nature Communications thanks Jan-Willem de Gier and the other, anonymous, reviewer(s) for their contribution to the peer review of this work

Reprints and permission information is available at http://www.nature.com/reprints

Publisher's note Springer Nature remains neutral with regard to jurisdictional claims in published maps and institutional affiliations.

Open Access This article is licensed under a Creative Commons Attribution 4.0 International License, which permits use, sharing, adaptation, distribution and reproduction in any medium or format, as long as you give appropriate credit to the original author(s) and the source, provide a link to the Creative Commons license, and indicate if changes were made. The images or other third party material in this article are included in the article's Creative Commons license, unless indicated otherwise in a credit line to the material. If material is not included in the article's Creative Commons license and your intended use is not permitted by statutory regulation or exceeds the permitted use, you will need to obtain permission directly from the copyright holder. To view a copy of this license, visit http://creativecommons.org/ licenses/by/4.0/

(C) The Author(s) 2021, corrected publication 2021 